\title{
On the cohomology mod $p$ of the classifying spaces of the exceptional Lie groups, I
}

\author{
By \\ Mamoru Mimura and Yuriko SAMBE \\ Dedicated to Professor A. Komatu on his 70-th birthday \\ (Received July 25, 1978)
}

\section{$\S 1$. Introduction}

Let $p$ be a prime and $G$ a compact, 1-connected simple Lie group. In general, when $H_{*}(G ; Z)$ has no $p$-torsion, the cohomology $\bmod p H^{*}\left(B G ; Z_{p}\right)$ of the classifying space $B G$ of $G$ is a polynomial algebra. When $H_{*}(G ; \boldsymbol{Z})$ has $p$-torsion, however, $H^{*}\left(B G ; \boldsymbol{Z}_{p}\right)$ is of complicated form.

Let $E_{i}$ be a compact, 1-connected exceptional Lie group of rank $i(i=6,7$, 8). Then $H_{*}\left(E_{i} ; Z\right)$ has $p$-torsion for $(i=6 ; p \leq 3),(i=7 ; p \leq 3)$ and $(i=8$; $p \leq 5)$. Of these the module structures of $H^{*}\left(B E_{i} ; Z_{2}\right)$ for $i=6,7$ have already been determined in [5] and [9] respectively.

The purpose of this series of papers is to investigate the structures of $H^{*}\left(B E_{i} ; Z_{3}\right)$ for $i=6,7,8$ and also of $H^{*}\left(B E_{8} ; Z_{5}\right)$.

Let $\{G: p\}$ be the set of all compact, associative $H$-spaces $X$ such that $H^{*}\left(X ; \boldsymbol{Z}_{p}\right) \cong H^{*}\left(G ; \boldsymbol{Z}_{p}\right)$ as Hopf algebras over the Steenrod algebra $\mathcal{A}_{p}$. (We do not necessarily assume the existence of a map between spaces inducing an isomorphism.) For every space $X$ of $\{G: p\}$ we have the EilenbergMoore spectral sequence $\left\{E_{r}, d_{r}\right\}$ such that

$$
\begin{aligned}
& E_{2} \cong \operatorname{Cotor}_{A}\left(\boldsymbol{Z}_{p}, \boldsymbol{Z}_{p}\right) \text { with } A=H^{*}\left(X ; \boldsymbol{Z}_{p}\right), \\
& E_{\infty} \cong \mathcal{G}_{2} H^{*}\left(B X ; \boldsymbol{Z}_{p}\right) .
\end{aligned}
$$

(Refer, for example, to [12] and [13] for the construction and the properties of the Eilenberg-Moore spectral sequence.)

In the present paper, Part I of the series, we determine the $E_{2}$-term of the Eilenberg-Moore spectral sequence for $X_{6}$ of $\left\{E_{6}: 3\right\}$ and for $X_{7}$ of $\left\{E_{7}: 3\right\}$. The main results are Theorems 4.10 and 5.20.

The paper is organized as follows. In $\S 2$ we construct an injective resolution of $\boldsymbol{Z}_{3}$ over $H^{*}\left(X_{7} ; \boldsymbol{Z}_{3}\right)$. In sections 3 and 4 we determine Cotor $_{A}$ $\left(\boldsymbol{Z}_{3}, \boldsymbol{Z}_{3}\right)$ for $A=H^{*}\left(X_{7} ; \boldsymbol{Z}_{3}\right)$. In the last section, $\S 5$, we construct an injective resolution of $\boldsymbol{Z}_{3}$ over $H^{*}\left(X_{6} ; \boldsymbol{Z}_{3}\right)$ and determine $\operatorname{Cotor}_{B}\left(\boldsymbol{Z}_{3}, \boldsymbol{Z}_{3}\right)$ for $B$ $=H^{*}\left(X_{6} ; \boldsymbol{Z}_{3}\right)$. The calculation in $\S 5$ is quite similar to but much simpler than 
that in $\S \S 3$ and 4 .

\section{§ 2. An injective resolution of $Z_{3}$ over $H^{*}\left(X_{7} ; Z_{3}\right)$}

First we recall the Hopf algebra structure of $H^{*}\left(X_{7} ; \boldsymbol{Z}_{3}\right)$ (over $\mathcal{A}_{3}$ ) from [7]:

(2. 1) As an algebra

$$
H^{*}\left(X_{7} ; Z_{3}\right) \cong Z_{3}\left[x_{8}\right] /\left(x_{8}^{3}\right) \otimes \Lambda\left(x_{3}, x_{7}, x_{11}, x_{15}, x_{19}, x_{27}, x_{35}\right),
$$

where deg $x_{i}=i$;

(2. 2) The coalgebra structure is given by

$$
\begin{array}{ll}
\bar{\phi}\left(x_{i}\right)=0 & \text { for } i=3,7,8,19, \\
\bar{\phi}\left(x_{j}\right)=x_{8} \otimes x_{j-8} & \text { for } j=11,15,27, \\
\bar{\phi}\left(x_{35}\right)=x_{8} \otimes x_{27}-x_{8}{ }^{2} \otimes x_{19}, &
\end{array}
$$

where $\bar{\phi}$ is the reduced diagonal map induced from the multiplication on $X_{7}$.

Notation. $A=H^{*}\left(X_{7} ; Z_{3}\right)$ and $\bar{A}=\tilde{H}^{*}\left(X_{7} ; Z_{3}\right)$.

We shall construct an injective resolution of $\boldsymbol{Z}_{\boldsymbol{3}}$ over $A$ using the same construction and the same notation as those in $\S 3$ of [8].

Take $L$ to be a graded $Z_{3}$-submodule of $\bar{A}$ generated by

$$
\left\{x_{3}, x_{7}, x_{8}, x_{19}, x_{11}, x_{15}, x_{27}, x_{8}^{2}, x_{35}\right\} \text {. }
$$

Let $\theta: A \rightarrow L$ be the projection and $\iota: L \rightarrow A$ the injection such that $\operatorname{co} \theta=1_{A}$. We name the set of corresponding elements under the suspension $s$ as

$$
s L=\left\{a_{4}, a_{8}, a_{9}, a_{20}, b_{12}, b_{18}, b_{28}, c_{17}, e_{30}\right\} .
$$

Define $\bar{\theta}: A \rightarrow s L$ by $\bar{\theta}=s \circ \theta$ and $\bar{\iota}: s L \rightarrow A$ by $\bar{\iota}=\iota \circ s^{-1}$. Let $T(s L)$ be the free tensor algebra over $s L$ with the (natural) product $\psi$. Consider the two sided ideal $I$ of $T(s L)$ generated by $\operatorname{Im}(\psi \circ(\bar{\theta} \otimes \bar{\theta}) \circ \phi)(\operatorname{Ker} \bar{\theta})$, where $\phi$ is the diagonal map of $A$. Then $I$ is generated by

(2. 4) $[\alpha, \beta]$ for all pairs $(\alpha, \beta)$ of generators of $T(s L)$ except $\left(a_{9}, b_{j}\right)$ $(j=12,16,28),\left(a_{9}, e_{38}\right)$ and $\left(a_{9}, c_{17}\right)$,

$$
\begin{aligned}
& {\left[a_{9}, b_{j}\right]+c_{17} a_{j-8} \text { for } j=12,16,28,} \\
& {\left[a_{9}, e_{36}\right]+c_{17} b_{28},}
\end{aligned}
$$

where $[\alpha, \beta]=\alpha \beta-(-1)^{*} \beta \alpha$ with $*=\operatorname{deg} \alpha \cdot \operatorname{deg} \beta$. 
Put $\bar{W}=T(s L) / I$, that is, $\bar{W}=Z_{3}\left\{a_{4}, a_{8}, a_{9}, a_{20}, b_{12}, b_{18}, b_{28}, c_{17}, e_{36}\right\}$. Note that $\bar{W}$ contains the polynomial algebra

$$
R=Z_{3}\left[a_{4}, a_{8}, a_{20}, b_{12}, b_{16}, b_{28}, e_{38}\right] .
$$

We define a map

$$
d=-\psi \circ(\bar{\theta} \otimes \bar{\theta}) \circ \phi \circ \bar{\iota}: \quad s L \rightarrow T(s L)
$$

and extend it naturally over $T(s L)$ as derivation. Since $d(I) \subset I$ holds, $d$ induces a map $\bar{W} \rightarrow \bar{W}$, which is again denoted by $d: \bar{W} \rightarrow \bar{W}$ by abuse of notation. It is easy to check that $d \circ d=0$ and so $\bar{W}$ is a differential algebra over $\boldsymbol{Z}_{3}$. Using the relation

$$
d \circ \bar{\theta}+\psi \circ(\bar{\theta} \otimes \bar{\theta}) \circ \phi=0,
$$

we can construct the twisted tensor product $W=A \otimes \bar{W}$ with respect to $\bar{\theta}$ [14]. Namely, $W$ is an $A$-comodule with the differential operator

$$
\bar{d}=1 \otimes d+(1 \otimes \psi) \circ(1 \otimes \bar{\theta} \otimes 1) \circ(\phi \otimes 1) .
$$

More explicitly, the differential operators $\bar{d}$ and $d$ are given by

$$
\begin{array}{ll}
\bar{d}\left(x_{i} \otimes 1\right)=1 \otimes a_{i+1} & \text { for } i=3,7,8,19, \\
\bar{d}\left(x_{8}{ }^{2} \otimes 1\right)=1 \otimes c_{17}-x_{8} \otimes a_{9}, & \\
\bar{d}\left(x_{j} \otimes 1\right)=1 \otimes b_{j+1}+x_{8} \bigotimes a_{j-7} & \text { for } j=11,15,27, \\
\bar{d}\left(x_{35} \otimes 1\right)=1 \otimes e_{38}+x_{8} \otimes b_{28}-x_{8}{ }^{2} \otimes a_{20} ; \\
d a_{i}=0 & \text { for } i=4,8,9,20, \\
d c_{17}=a_{9}{ }^{2}, & \\
d b_{j}=-a_{9} a_{j-8} & \text { for } j=12,16,28, \\
d e_{36}=-a_{9} b_{28}+c_{17} a_{20} . &
\end{array}
$$

Now we define weight in $W=A \otimes \bar{W}$ as follows:

$$
\begin{array}{rccccccccc}
A: & x_{3}, & x_{7}, & x_{19}, & x_{8}, & x_{8}{ }^{2}, & x_{11}, & x_{15}, & x_{27}, & x_{35} \\
\bar{W}: & a_{4}, & a_{8}, & a_{20}, & a_{9}, & c_{17}, & b_{12}, & b_{18}, & b_{28}, & e_{36} \\
\text { weight: } & 0 & 0 & 0 & 1 & 2 & 2 & 2 & 2 & 6
\end{array}
$$

(The weight of a monomial is the sum of the weights of each element.) Define a filtration

$$
F_{r}=\{x \mid \text { weight } x \leq r\} .
$$

Put $E_{0} W=\sum F_{i} / F_{i-1}$. Then it is easy to see that

$$
E_{0} W \cong \Lambda\left(x_{3}, x_{7}, x_{19}, x_{11}, x_{15}, x_{27}, x_{35}\right) \otimes Z_{3}\left[a_{4}, a_{8}, a_{20}, b_{12}, b_{16}, b_{28}, e_{36}\right] \otimes C\left(Q\left(x_{8}\right)\right),
$$


where $C\left(Q\left(x_{8}\right)\right)$ is the cobar construction of $Z_{3}\left[x_{8}\right] /\left(x_{8}{ }^{3}\right)$. The differential formulae (2.5) and (2.6) imply that $E_{0} W$ is acylic, and hence $W$ is acyclic.

Theorem 2.9. $W$ is an injective resolution of $Z_{3}$ over $A=H^{*}\left(X_{7}\right.$; $\left.Z_{3}\right)$.

By the definition of Cotor we have

Corollary 2. 10. $H(\bar{W}: d)=\operatorname{Ker} d / \operatorname{Im} d \cong \operatorname{Cotor}_{A}\left(\boldsymbol{Z}_{3}, \boldsymbol{Z}_{3}\right)$.

\section{$\S 3$. Elements with neither $a_{9}$ nor $c_{17}$ in $\operatorname{Cotor}_{A}\left(Z_{3}, Z_{3}\right)$}

We define an operator $\partial$ by

$$
\begin{array}{ll}
\partial a_{i}=0 & \text { for } i=4,8,20, \\
\partial b_{j}=-a_{j-8} & \text { for } j=12,16,28, \\
\partial e_{38}=-b_{28}, &
\end{array}
$$

and extend it over $R=Z_{3}\left[a_{4}, a_{8}, a_{20}, b_{12}, b_{18}, b_{28}, e_{38}\right]$ so that it satisfies

$$
\begin{aligned}
\partial(P+Q)=\partial P+\partial Q \text { and } & \partial(P Q)=\partial P \cdot Q+P \cdot \partial Q \\
& \text { for any polynomials } P \text { and } Q .
\end{aligned}
$$

Then we have

Lemma 3. 3. For a polynomial $P \in R$ we have

$$
\begin{aligned}
& \partial^{3} P=0, \\
& {\left[a_{9}, P\right]=c_{17} \partial P,} \\
& d P=a_{9} \partial P+c_{17} \partial^{2} P .
\end{aligned}
$$

Proof. (By induction.) Suppose that $\partial^{3} P=0$ holds for any polynomial $P$ of degree up to $l$. Then

$$
\partial^{3}(x P)=\partial^{3} x \cdot P+x \cdot \partial^{3} P=0 .
$$

Thus $\partial^{3} P=0$ holds for a polynomial of degree $l+1$.

Suppose that $\left[a_{9}, P\right]=c_{17} \partial P$ holds for any polynomial $P$ of degree up to l. Then

$$
\left[a_{9}, x P\right]=\left[a_{9}, x\right] P+x\left[a_{9}, P\right]=c_{17} \partial x \cdot P+x c_{17} \partial P=c_{17} \partial(x P) .
$$

Thus the relation holds for a polynomial of degree $l+1$. 
Suppose that $d P=a_{9} \partial P+c_{17} \partial^{2} P$ holds for any polynomial of degree up to l. Then

$$
\begin{aligned}
d(x P) & =d x \cdot P+x \cdot d P \\
& =\left(a_{9} \partial x+c_{17} \partial^{2} x\right) P+x\left(a_{9} \partial P+c_{17} \partial^{2} P\right) \\
& =a_{9} \partial x \cdot P+c_{17} \partial^{2} x \cdot P+\left(a_{9} x-c_{17} \partial x\right) \partial P+c_{17} x \partial^{2} P \\
& =a_{9} \partial(x P)+c_{17} \partial^{2}(x P) .
\end{aligned}
$$

Thus the differential formula holds for a polynomial of degree $l+1$. q.e.d.

Lemma 3. 4. Let $P$ be non-trivial in $R$. Then $P$ is a non-trivial cocycle if and only if $\partial P=0$.

Proof. If $P$ is a cocycle, $d P=0$. Then by the differential formula, we have $\partial P=0$.

Conversely, if $\partial P=0$, so does $\partial^{2} P$, whence we have $d P=0$ by the differential formula. Since $P$ contains no $a_{9}$, it is not in the $d$-image, hence it is a non-trivial cocycle.

q.e.d.

We shall find cocycles in the following steps:

(i) cocycles in $\boldsymbol{Z}_{3}\left[a_{4}, a_{8}, a_{20}, b_{12}, b_{16}\right]$,

(ii) those in $Z_{3}\left[a_{4}, a_{8}, a_{20}, b_{12}, b_{16}, b_{28}\right]$,

(iii) those in $Z_{3}\left[a_{4}, a_{8}, a_{20}, b_{12}, b_{18}, b_{28}, e_{38}\right]$,

(iv) those in $Z_{3}\left\{a_{9}, c_{17}\right\}$,

(v) other cocycles.

(The last two steps will be done in $\S 4$.)

(i) Cocycles in $Z_{3}\left[a_{4}, a_{8}, a_{20}, b_{12}, b_{16}\right]$

Clearly, $a_{4}, a_{8}$ and $a_{20}$ are cocycles.

A cocycle of degree 1 with respect to $b_{12}$ and $b_{16}$ is of the form $P=A b_{12}$ $+B b_{16}$ with $A, B \in Z_{3}\left[a_{4}, a_{8}, a_{20}\right]$. The relation $\partial P=-A a_{4}-B a_{8}=0$ yields an indecomposable cocycle

$$
y_{20}=a_{8} b_{12}-a_{4} b_{10} .
$$

A cocycle of degree 2 with respect to $b_{12}$ and $b_{16}$ is of the form

$$
P=A b_{12}{ }^{2}+B b_{18}{ }^{2}+C b_{12} b_{10} \text { with } A, B, C \in Z_{3}\left[a_{4}, a_{8}, a_{20}\right] .
$$

Then $\partial P=\left(A a_{4}-C a_{8}\right) b_{12}+\left(B a_{8}-C a_{4}\right) b_{16}=0$ gives rise to 


$$
A a_{4}-C a_{8}=0 \text { and } B a_{8}-C a_{4}=0,
$$

from which we obtain a decomposable cocycle

$$
P=a_{8}^{2} b_{12}{ }^{2}+a_{4}^{2} b_{16}{ }^{2}+a_{4} a_{8} b_{12} b_{18}=y_{20}^{2} \text {. }
$$

A cocycle of degree 3 with respect to $b_{12}$ and $b_{18}$ is of the form

$$
P=A b_{12}{ }^{3}+B b_{18}{ }^{3}+C b_{12}{ }^{2} b_{18}+D b_{12} b_{18}{ }^{2} \text {. }
$$

Then $\partial P=-C a_{8} b_{12}{ }^{2}+\left(C a_{4}+D a_{8}\right) b_{12} b_{16}-D a_{4} b_{18}{ }^{2}=0$ gives rise to $C=D=0$. Thus we have two new cocycles

$$
x_{38}=b_{12}{ }^{3} \text { and } x_{48}=b_{18}{ }^{3} .
$$

A cocycle of degree 4 is of the form

$$
P=b_{12}{ }^{3}\left(A b_{12}+B b_{16}\right)+C b_{12}{ }^{2} b_{18}{ }^{2}+b_{16}{ }^{3}\left(D b_{12}+E b_{16}\right) \text {. }
$$

Then $\partial P=0$ implies that $C=0$, since no term with $b_{12} b_{18}{ }^{2}$ appears except for $C a_{4} b_{12} b_{16}{ }^{2}$. Further $\partial P=0$ gives rise to $\partial\left(A b_{12}+B b_{16}\right)=\partial\left(D b_{12}+E b_{18}\right)=0$, that is, $P$ is decomposed in cocycles $b_{12}{ }^{3}, b_{18}{ }^{3}, A b_{12}+B b_{16}$ and $D b_{12}+E b_{16}$. So no new cocycles are obtained.

Similarly, any cocycles of degree higher than 4 is decomposable.

We have obtained

Result (i). The following are all the indecomposable cocycles in $Z_{3}$ $\left[a_{4}, a_{8}, a_{20}, b_{12}, b_{16}\right]$ :

$$
a_{4}, \quad a_{8}, \quad a_{20}, \quad y_{20}\left(=a_{8} b_{12}-a_{4} b_{10}\right), \quad x_{36}\left(=b_{12}{ }^{3}\right), \quad x_{48}\left(=b_{16}{ }^{3}\right) .
$$

\section{(ii) Cocycles in $Z_{3}\left[a_{4}, a_{8}, a_{20}, b_{12}, b_{18}, b_{28}\right]$}

A cocycle of degree 1 with respect to $b_{28}$ is of the form $P=A b_{28}+B$ with $A, B \in \boldsymbol{Z}_{3}\left[a_{4}, a_{8}, a_{20}, b_{12}, b_{18}\right]$. Then $\partial P=\partial A \cdot b_{28}-a_{20} A+\partial B=0$ gives rise to

$$
\partial A=0 \text { and } \partial B=a_{20} A .
$$

So by Lemma 3. 4, $A$ is a cocycle and thus an element in $Z_{3}\left[a_{4}, a_{8}, a_{20}\right.$, $\left.y_{20}, x_{38}, x_{48}\right]$, for which we have to find, if any, a $B$ such that $\partial B=a_{20} A$. Note that it is sufficient to choose one such $B$, since the difference of two cocyles $P=A b_{28}+B$ and $P^{\prime}=A b_{28}+B^{\prime}$ is a cocycle without $b_{28}$ :

$$
P-P^{\prime}=\left(A b_{28}+B\right)-\left(A b_{28}+B^{\prime}\right)=B-B^{\prime} .
$$

Note also that, if there is a cocyle $P_{i}$ corresponding to $A_{i}: P_{i}=A_{i} b_{28}+\cdots$ $(i=1,2)$, then cocycles corresponding to the sum $A_{1}+A_{2}$ and to the product $A_{1} A_{2}$ exist and are decomposable: 


$$
\begin{aligned}
& P_{1}+P_{2}=\left(A_{1}+A_{2}\right) b_{28}+\cdots, \\
& A_{1} P_{2}=A_{1} A_{2} b_{28}+\cdots .
\end{aligned}
$$

Now $A$ is an element in $Z_{3}\left[a_{4}, a_{8}, a_{20}, y_{20}, x_{38}, x_{48}\right]$. In particular, for $A$ $=a_{4}, a_{8}$ and $y_{20}{ }^{2}\left(=\partial^{2}\left(-b_{12}{ }^{2} b_{18}{ }^{2}\right)\right)$, we can choose $B=-a_{20} b_{12},-a_{20} b_{18}$ and $a_{20} \partial$ $\left(-b_{12}{ }^{2} b_{10}{ }^{2}\right)$ respectively, and we have corresponding cocycles

$$
\begin{aligned}
y_{32} & =a_{4} b_{28}-a_{20} b_{12}, \\
y_{38} & =a_{8} b_{28}-a_{20} b_{18}, \\
-y_{88} & =\partial^{2}\left(-b_{12}{ }^{2} b_{18}{ }^{2}\right) b_{28}+a_{20} \partial\left(-b_{12}{ }^{2} b_{18}{ }^{2}\right)=-\partial^{2}\left(b_{12}{ }^{2} b_{18}{ }^{2} b_{28}\right) .
\end{aligned}
$$

Thus for $A=a_{4} A^{\prime}+a_{8} A^{\prime \prime}+y_{20}{ }^{2} A^{\prime \prime \prime}$, we have a decomposable cocycle $P=y_{32} A^{\prime}$ $+y_{38} A^{\prime \prime}-y_{88} A^{\prime \prime}$.

A monomial in cocycles for $A$ that has no $a_{4}, a_{3}$ nor $y_{20}{ }^{2}$ is of the form $a_{20}{ }^{i} x_{38}{ }^{j} x_{48}{ }^{k}$ or $\mathrm{y}_{20} a_{20}{ }^{i} x_{38}{ }^{j} x_{48}{ }^{k}$ (where $i, j, k$ are non-negative integers), for which there is no $B$ satisfying the conditions. Neither is there $B$ for $A=a_{20}{ }^{i} x_{36}{ }^{j} x_{48}{ }^{k}$ $+A^{\prime}$ and $y_{20} a_{20}{ }^{i} x_{38}{ }^{j} x_{48}{ }^{k}+A^{\prime}$ whatever a cocycle in $Z_{3}\left[a_{4}, a_{8}, a_{20}, y_{20}, x_{38}, x_{48}\right] A^{\prime}$ is.

We have thus

(3. 5) The indecomposable cocycles of degree 1 with respect to $b_{28}$ are $y_{32}, y_{36}$ and $y_{68}$.

A cocycle of degree 2 with respect to $b_{28}$ is of the form $P=A b_{28}{ }^{2}+B b_{28}$ $+C$ with $A, B, C \in Z_{3}\left[a_{4}, a_{8}, a_{20}, b_{12}, b_{16}\right]$. Then the relation

$$
\partial P=\partial A \cdot b_{28}^{2}+\left(a_{20} A+\partial B\right) b_{28}+\left(-a_{20} B+\partial C\right)=0
$$

gives rise to

$$
\partial A=0, \partial B=-a_{20} A \text { and } \partial C=a_{20} B .
$$

Again by Lemma 3. 4, $A$ is a cocycle, that is, an element in $Z_{3}\left[a_{4}, a_{8}, a_{20}, y_{20}\right.$, $\left.x_{38}, x_{48}\right]$. The difference of two cocycles $P=A b_{28}{ }^{2}+B b_{28}+C$ and $P^{\prime}=A b_{28}{ }^{2}$ $+B^{\prime} b_{28}+C^{\prime}$ is a cocycle $\left(B-B^{\prime}\right) b_{28}+\left(C-C^{\prime}\right)$, that is, a cocycle of lower degree with respect to $b_{28}$. So it is sufficient to choose, if any, one corresponding cocycle for a cocycle $A$.

Once again, if there is a cocycle $P_{i}$ corresponding to $A_{i}(i=1,2)$, then there exist cocycles corresponding to $A_{1}+A_{2}$ and to $A_{1} A_{2}$, which are decomposable.

For a cocycle $A$ of the form $\partial^{2} D$ with $D \in Z_{3}\left[a_{4}, a_{8}, a_{20}, b_{12}, b_{16}\right]$, we have actually a corresponding cocycle $P=\partial^{2}\left(D b_{28}{ }^{2}\right)$. So we have a cocycle for each of the following:

$$
a_{4}{ }^{2}=\partial^{2}\left(-b_{12}{ }^{2}\right), a_{8}{ }^{2}=\partial^{2}\left(-b_{18}{ }^{2}\right), a_{4} a_{8}=\partial^{2}\left(-b_{12} b_{18}\right),
$$




$$
a_{4} y_{20}=\partial^{2}\left(b_{12}{ }^{2} b_{16}\right), a_{8} y_{20}=\partial^{2}\left(-b_{12} b_{16}{ }^{2}\right), y_{20}{ }^{2}=\partial^{2}\left(-b_{12}{ }^{2} b_{10}{ }^{2}\right) \text {. }
$$

Cocycles $P=\partial^{2}\left(-b_{12}{ }^{2} b_{28}{ }^{2}\right), \partial^{2}\left(-b_{18}{ }^{2} b_{28}{ }^{2}\right)$ and $\partial^{2}\left(-b_{12} b_{18} b_{28}{ }^{2}\right)$ corresponding respectively to $a_{4}{ }^{2}, a_{8}{ }^{2}$ and $a_{4} a_{8}$ are $y_{32}{ }^{2}, y_{36}{ }^{2}$ and $y_{32} y_{38}$ respectively, and hence they are decomposable.

Now we put

$$
\begin{aligned}
& y_{80}=\partial^{2}\left(b_{12}{ }^{2} b_{18} b_{28}{ }^{2}\right)=a_{4} y_{20} b_{28}{ }^{2}+\cdots, \\
& y_{84}=\partial^{2}\left(b_{12} b_{18}{ }^{2} b_{28}{ }^{2}\right)=-a_{8} y_{20} b_{28}{ }^{2}+\cdots, \\
& y_{96}=\partial^{2}\left(b_{12}{ }^{2} b_{18}{ }^{2} b_{28}{ }^{2}\right)=-y_{20}{ }^{2} b_{28}{ }^{2}+\cdots .
\end{aligned}
$$

Lemma 3. 7. The cocycles $y_{80}, y_{84}$ and $y_{98}$ are indecomposable and there is no other indecomposable cocycle of degree 2 with respect to $b_{28}$.

Proof. First we study $A$ in $Z_{3}\left[a_{4}, a_{8}, y_{20}\right]$. For $A=a_{4}$, we have $B$ $=a_{20} b_{12}+(\partial$-kernel $)$, but no $C$ in $Z_{3}\left[a_{4}, a_{8}, a_{20}, b_{12}, b_{16}\right]$ such that $\partial C=a_{20}{ }^{2} b_{12}$ + (other terms), thus there is no cocycle beginning with $a_{4} b_{28}{ }^{2}$. Similarly, there is none beginning with $a_{8} b_{28}{ }^{2}$. For $A=y_{20}$, there is no $B$ in $Z_{3}\left[a_{4}, a_{8}, a_{20}, b_{12}\right.$, $\left.b_{16}\right]$ such that $\partial B=-a_{20} y_{20}$, and so there is no cocycle that begins with $y_{20} b_{28}{ }^{2}$. Recall that there is also no cocycle that begins with $y_{20} b_{28}$. And we also see that there is no cocycle beginning with $A b_{28}{ }^{2}$ whatever a sum of $a_{4}, a_{8}$ and $y_{20} A$ is. We conclude that the cocycles $y_{80}, y_{84}$ and $y_{96}$ are indecomposable.

We have seen that each monomial of degree 2 in $a_{4}, a_{8}$ and $y_{20}$ has a corresponding cocycle (decomposable or indecomposable). Therefore any polynomial $A$ in $a_{4}, a_{8}$ and $y_{20}$ of degree higher or equal to 2 has a corresponding cocycle, which is decomposable except for $y_{80}, y_{84}$ and $y_{96}$. Thus there is no other indecomposable cocycle for $A \in Z_{3}\left[a_{4}, a_{8}, y_{20}\right]$.

Now we consider cocycles in $\boldsymbol{Z}_{3}\left[a_{4}, a_{8}, a_{20}, y_{20}, x_{38}, x_{48}\right] \cong \boldsymbol{Z}_{3}\left[a_{4}, a_{8}, y_{20}\right] \otimes \boldsymbol{Z}_{3}$ $\left[a_{20}, x_{36}, x_{48}\right]$.

As we have noted, there exists a cocycle corresponding to $A a_{20}{ }^{i} x_{36}{ }^{j} x_{48}{ }^{k}$ provided there is a cocycle corresponsing to $A$ (here in particular, to $A \in$ $Z_{3}\left[a_{4}, a_{8}, y_{20}\right]$ ), although it is decomposable.

Since $a_{20}, b_{12}$ and $b_{18}$ are not in the image $\partial\left(\boldsymbol{Z}_{3}\left[a_{4}, a_{8}, b_{12}, b_{16}\right]\right)$ and since $\partial a_{20}$ $=\partial x_{36}=\partial x_{48}=0$, the elements $a_{20}, x_{36}$ and $x_{48}$ are 'immobile' under $\partial$ when seeking $B$ or $C$. It follows that there is no cocycle corresponding to $A a_{20}{ }^{i} x_{38}{ }^{j}$ $x_{48}{ }^{k}+$ (other cocycles) if there is none corresponding to $A$. Therefore there is no other indecomposable cocycle of degree 2 with respect to $b_{28}$. q.e.d.

Finally, $x_{84}=b_{28}{ }^{3}$ is the only indecomposable cocycle of degree 3 with respect to $b_{28}$. It is easy to see that there is no new cocycle of degree higher than 3 .

We have 
Result (ii). The following are all the indecomposable cocycles in $Z_{3}\left[a_{4}, a_{8}, a_{20}, b_{12}, b_{16}, b_{28}\right]$ :

$$
\begin{aligned}
& a_{4}, \quad a_{8}, \quad a_{20}=\partial^{2} e_{38}, \\
& x_{38}=b_{12}{ }^{3}, \quad x_{18}=b_{18}{ }^{3}, \quad x_{84}=b_{28}{ }^{3}=\partial^{2}\left(-b_{28} e_{36}{ }^{2}\right), \\
& y_{20}=a_{8} b_{12}-a_{4} b_{18}, \\
& y_{32}=a_{4} b_{28}-a_{20} b_{12}=\partial^{2}\left(-b_{12} e_{36}\right), \\
& y_{38}=a_{8} b_{28}-a_{20} b_{18}=\partial^{2}\left(-b_{18} e_{36}\right), \\
& y_{68}=\partial^{2}\left(b_{12}{ }^{2} b_{18}{ }^{2} b_{28}\right)=y_{20}{ }^{2} b_{28}+\cdots, \\
& y_{80}=\partial^{2}\left(b_{12}{ }^{2} b_{18} b_{28}{ }^{2}\right)=a_{4} y_{20} b_{28}{ }^{2}+\cdots, \\
& y_{84}=\partial^{2}\left(b_{12} b_{18}{ }^{2} b_{28}{ }^{2}\right)=-a_{8} y_{20} b_{28}{ }^{2}+\cdots, \\
& y_{96}=\partial^{2}\left(b_{12}{ }^{2} b_{18}{ }^{2} b_{28}{ }^{2}\right)=-y_{20}{ }^{2} b_{28}{ }^{2}+\cdots .
\end{aligned}
$$

(Result (i) is included in Result (ii).)

The following will be needed in the calculation in step (iii).

Lemma 3. 8. The elements $y_{68}, y_{80}, y_{84}, y_{98}$ and the following elements appear in the image $\partial^{2}\left(\boldsymbol{Z}_{3}\left[a_{4}, a_{8}, a_{20}, b_{12}, b_{16}, b_{28}\right]\right)$ :

$$
\begin{aligned}
& a_{4}{ }^{2}=\partial^{2}\left(-b_{12}{ }^{2}\right), \quad a_{8}{ }^{2}=\partial^{2}\left(-b_{18}{ }^{2}\right), \quad a_{20}{ }^{2}=\partial^{2}\left(-b_{28}{ }^{2}\right), \\
& a_{4} a_{8}=\partial^{2}\left(-b_{12} b_{18}\right), \quad a_{4} a_{20}=\partial^{2}\left(-b_{12} b_{28}\right), \quad a_{8} a_{20}=\partial^{2}\left(-b_{16} b_{28}\right), \\
& a_{4} y_{20}=\partial^{2}\left(b_{12}{ }^{2} b_{16}\right), \quad a_{8} y_{20}=\partial^{2}\left(-b_{12} b_{18}{ }^{2}\right), \\
& a_{4} y_{32}=\partial^{2}\left(-b_{12}{ }^{2} b_{28}\right), \quad a_{20} y_{32}=\partial^{2}\left(b_{12} b_{28}{ }^{2}\right) \text {, } \\
& a_{8} y_{36}=\partial^{2}\left(-b_{18}{ }^{2} b_{28}\right), \quad a_{20} y_{38}=\partial^{2}\left(b_{18} b_{28}{ }^{2}\right) \text {, } \\
& a_{20} y_{20}-a_{8} y_{32}=-a_{20} y_{20}-a_{4} y_{36}=a_{4} y_{36}+a_{8} y_{32}=\partial^{2}\left(b_{12} b_{18} b_{28}\right) \text {, } \\
& y_{20}{ }^{2}=\partial^{2}\left(-b_{12}{ }^{2} b_{18}{ }^{2}\right), \quad y_{32}{ }^{2}=\partial^{2}\left(-b_{12}{ }^{2} b_{28}{ }^{2}\right), \quad y_{38}{ }^{2}=\partial^{2}\left(-b_{18}{ }^{2} b_{28}{ }^{2}\right) \text {, } \\
& y_{20} y_{32}=\partial^{2}\left(b_{12}{ }^{2} b_{18} b_{28}\right), \quad y_{20} y_{36}=\partial^{2}\left(-b_{12} b_{18}{ }^{2} b_{28}\right) \text {, } \\
& y_{32} y_{36}=\partial^{2}\left(-b_{12} b_{18} b_{28}{ }^{2}\right) \text {, }
\end{aligned}
$$

and $P \cdot \partial^{2} Q=\partial^{2}(P \cdot Q)$ for any cocycle $P$ and any polynomial $Q$.

Proof is by direct calculation.

\section{(iii) Cocycles in $Z_{3}\left[a_{4}, a_{8}, a_{20}, b_{12}, b_{16}, b_{28}, e_{38}\right]$}

A cocycle of degree 1 with respect to $e_{38}$ is of the form $P=A e_{36}+B$ with $A, B \in Z_{3}\left[a_{4}, a_{8}, a_{20}, b_{12}, b_{18}, b_{28}\right]$. Then $\partial P=\partial A \cdot e_{38}-A b_{28}+\partial B=0$ gives rise to

$$
\partial A=0 \quad \text { and } \quad A b_{28}=\partial B \text {. }
$$


Thus $A$ is a cocycle, for which it is sufficient to find, if any, one corresponding cocycle just as in (ii). For a cocycle $A$ of the form $\partial^{2} C$ with $C \in \boldsymbol{Z}_{3}\left[a_{4}, a_{8}, a_{20}, b_{12}, b_{16}, b_{28}\right]$, we have actually a corresponding cocycle $P=\partial^{2}$ $\left(C e_{38}\right)$ and for $A$ of the form $\partial^{2}\left(C e_{36}\right)$ with $C$ as above, we have a corresponding cocycle $P=\partial^{2}\left(-C e_{38}{ }^{2}\right)$.

Result (iii-1). We have the following indecomposable cocycles of degree 1 with respect to $e_{38}$ :

$$
\begin{aligned}
& z_{58}=\partial^{2}\left(e_{36}{ }^{2}\right)=-a_{20} e_{36}+\cdots, \\
& z_{44}=\partial^{2}\left(b_{12}{ }^{2} e_{36}\right)=-a_{4}{ }^{2} e_{36}+\cdots, \\
& z_{48}=\partial^{2}\left(b_{12} b_{18} e_{36}\right)=-a_{4} a_{8} e_{36}+\cdots, \\
& z_{52}=\partial^{2}\left(b_{16}{ }^{2} e_{36}\right)=-a_{8}{ }^{2} e_{36}+\cdots, \\
& z_{68}=\partial^{2}\left(b_{12} e_{36}{ }^{2}\right)=y_{32} e_{36}+\cdots, \\
& z_{72}=\partial^{2}\left(b_{16} e_{36}{ }^{2}\right)=y_{38} e_{36}+\cdots, \\
& z_{60}=\partial^{2}\left(b_{12}{ }^{2} b_{18} e_{36}\right)=a_{4} y_{20} e_{36}+\cdots, \\
& z_{64}=\partial^{2}\left(b_{12} b_{16}{ }^{2} e_{38}\right)=-a_{8} y_{20} e_{38}+\cdots, \\
& z_{76}=\partial^{2}\left(b_{12}{ }^{2} b_{16}{ }^{2} e_{36}\right)=-y_{20}{ }^{2} e_{36}+\cdots, \\
& z_{104}=\partial^{2}\left(b_{12}{ }^{2} b_{18}{ }^{2} b_{28} e_{36}\right)=y_{88} e_{36}+\cdots, \\
& z_{116}=\partial^{2}\left(b_{12}{ }^{2} b_{16} b_{28}{ }^{2} e_{36}\right)=y_{80} e_{36}+\cdots, \\
& z_{120}=\partial^{2}\left(b_{12} b_{16}{ }^{2} b_{28}{ }^{2} e_{36}\right)=y_{84} e_{36}+\cdots, \\
& z_{132}=\partial^{2}\left(b_{12}{ }^{2} b_{18}{ }^{2} b_{28}{ }^{2} e_{36}\right)=y_{98} e_{36}+\cdots,
\end{aligned}
$$

Proof. We have indecomposable cocycles $-z_{58}, z_{68}, z_{72}, z_{104}, z_{118}, z_{120}$ and $z_{132}$ corresponding respectively to $a_{20}, y_{32}, y_{36}, y_{68}, y_{80}, y_{84}$ and $y_{96}$. Therefore, if each term of a cocycle $A$ contains one of $a_{20}, y_{32}, y_{36}, y_{88}, y_{80}, y_{84}$ or $y_{96}$, a cocycle beginning with $A e_{36}$ is decomposable.

Cocycles that we have to consider next as $\mathrm{A}$ are polynomials in $a_{4}, a_{8}, y_{20}$, $x_{36}, x_{48}$ and $x_{84}$. Recall that $x_{36}$ and $x_{48}$ as well as $x_{84}=b_{28}{ }^{3}$ are 'immobile' under $\partial$ as before and so there is no cocycle corresponding to $A x_{38}{ }^{i} x_{48}{ }^{j} x_{84}{ }^{k}$ + (other cocycles) if there is none corresponding to $A$. In particular, we have none corresponding to $x_{88}{ }^{i} x_{48}{ }^{j} x_{84}{ }^{k}$.

We have now only to consider those $A$ in $\boldsymbol{Z}_{3}\left[a_{4}, a_{8}, y_{20}\right]$.

For $A$ a sum of $a_{4}, a_{8}$ and $y_{20}$, there is no $B$ satisfying $A b_{28}=\partial B$, whence there is no cocycle that begins with $a_{4} e_{38}, a_{8} e_{36}$ or $y_{20} e_{36}$. So the cocycles corresponding to $a_{4}{ }^{2}, a_{4} a_{8}, a_{8}{ }^{2}, a_{4} y_{20}, a_{8} y_{20}$ and $y_{20}{ }^{2}$ are all indecomposable. Any monomial $A$ in $a_{4}, a_{8}$ and $y_{20}$ of degree higher than 2 has one of $a_{4}{ }^{2}, a_{4} a_{8}, a_{3}^{2}$, $a_{4} y_{20}, a_{8} y_{20}$ or $y_{20}{ }^{2}$, whence any cocycle corresponding to such $A$ is decomposable. We have shown that the cocycles in (iii-1) are all the indecomposable 
ones of degree 1 with respect to $e_{38}$.

q.e.d.

A cocycle of degree 2 with respect to $e_{38}$ is of the form $P=A e_{36}{ }^{2}+B e_{36}$ $+C$ with $A, B, C \in Z_{3}\left[a_{4}, a_{8}, a_{20}, b_{12}, b_{18}, b_{28}\right]$. Then $\partial P=0$ gives rise to

$$
\partial A=0, \quad A b_{28}=-\partial B \text { and } B b_{28}=\partial C \text {. }
$$

Therefore $A$ is a cocycle, for which it is again sufficient to find, if any, one corresponding cocycle. We have actually

(3.9) There is a cocycle $P=\partial^{2}\left(D e_{36}{ }^{2}\right)$ corresponding to a cocycle $A$ of the form $\partial^{2} D$ with $D \in Z_{3}\left[a_{4}, a_{8}, a_{20}, b_{12}, b_{18}, b_{28}\right]$.

Result (iii-2). The following are the indecomposable cocycles of degree 2 with respect to $e_{38}$ :

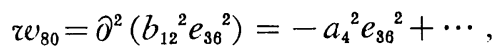

$$
\begin{aligned}
& w_{84}=\partial^{2}\left(b_{12} b_{18} e_{38}{ }^{2}\right)=-a_{4} a_{8} e_{38}{ }^{2}+\cdots, \\
& w_{88}=\partial^{2}\left(b_{18}{ }^{2} e_{38}{ }^{2}\right)=-a_{8}{ }^{2} e_{38}{ }^{2}+\cdots \text {, } \\
& w_{98}=\partial^{2}\left(b_{12} b_{28} e_{38}^{2}\right)=-a_{4} a_{20} e_{36}{ }^{2}+\cdots, \\
& w_{100}=\partial^{2}\left(b_{18} b_{28} e_{38}{ }^{2}\right)=-a_{8} a_{20} e_{30}{ }^{2}+\cdots, \\
& v_{98}=\partial^{2}\left(b_{12}{ }^{2} b_{18} e_{38}{ }^{2}\right)=a_{4} y_{20} e_{38}{ }^{2}+\cdots, \\
& v_{100}=\partial^{2}\left(b_{12} b_{18}{ }^{2} e_{38}^{2}\right)=-a_{8} y_{20} e_{36}{ }^{2}+\cdots \text {, } \\
& v_{108}=\partial^{2}\left(b_{12}{ }^{2} b_{28} e_{38}{ }^{2}\right)=-a_{4} y_{32} e_{38}{ }^{2}+\cdots, \\
& v_{112}=\partial^{2}\left(b_{12} b_{19} b_{28} e_{38}{ }^{2}\right)=\left(a_{20} y_{20}-a_{8} y_{32}\right) e_{38}{ }^{2}+\cdots \\
& =\left(-a_{20} y_{20}-a_{4} y_{36}\right) e_{38}{ }^{2}+\cdots \\
& =\left(a_{4} y_{36}+a_{8} y_{32}\right) e_{36}^{2}+\cdots, \\
& v_{118}=\partial^{2}\left(b_{18}{ }^{2} b_{28} e_{38}{ }^{2}\right)=-a_{8} y_{38} e_{38}{ }^{2}+\cdots \text {, } \\
& u_{112}=\partial^{2}\left(b_{12}{ }^{2} b_{10}{ }^{2} e_{38}{ }^{2}\right)=-y_{20}{ }^{2} e_{36}{ }^{2}+\cdots, \\
& u_{124}=\partial^{2}\left(b_{12}{ }^{2} b_{18} b_{28} e_{38}{ }^{2}\right)=y_{20} y_{32} e_{38}{ }^{2}+\cdots \text {, } \\
& u_{128}=\partial^{2}\left(b_{12} b_{18}{ }^{2} b_{28} e_{36}{ }^{2}\right)=-y_{20} y_{38} e_{38}{ }^{2}+\cdots, \\
& p_{140}=\partial^{2}\left(b_{12}{ }^{2} b_{18}{ }^{2} b_{28} e_{38}{ }^{2}\right)=y_{88} e_{38}{ }^{2}+\cdots \text {, } \\
& p_{152}=\partial^{2}\left(b_{12}{ }^{2} b_{18} b_{28}{ }^{2} e_{38}{ }^{2}\right)=y_{80} e_{38}{ }^{2}+\cdots \text {, } \\
& p_{156}=\partial^{2}\left(b_{12} b_{18}{ }^{2} b_{28}{ }^{2} e_{38}{ }^{2}\right)=y_{84} e_{38}{ }^{2}+\cdots \text {, } \\
& p_{188}=\partial^{2}\left(b_{12}{ }^{2} b_{18}{ }^{2} b_{28}{ }^{2} e_{38}{ }^{2}\right)=y_{98} e_{38}{ }^{2}+\cdots \text {. }
\end{aligned}
$$

Proof. By virtue of (3.9) we have 23 cocycles corresponding to the 23 
elements in Lemma 3.8. Of these cocycles 17 are indecomposable and the 6 corresponding to $a_{20}{ }^{2}, a_{20} y_{32}, a_{20} y_{36}, y_{32}{ }^{2}, y_{36}{ }^{2}$ and $y_{32} y_{36}$ are decomposable.

The cocycles $p_{140}, p_{152}, p_{156}$ and $p_{168}$ corresponding respectively to $y_{68}, y_{80}, y_{84}$ and $y_{96}$ are indecomposable, and any cocycle which corresponds to a monomial $A$ having one of $y_{68}, y_{80}, y_{84}$ and $y_{96}$ is decomposable.

Note again that there is no cocycle corresponding to $A x_{38}{ }^{i} x_{48}{ }^{j} x_{84}{ }^{k}+$ (other cocycles) if there is none corresponding to $A$. In particular, there is no cocycle corresponding to $x_{36}{ }^{i} x_{48}{ }^{3} x_{84}{ }^{k}$. Thus we have only to consider polynomials in $a_{4}, a_{8}, a_{20}, y_{20}, y_{32}$ and $y_{36}$ as $A$.

For $A=a_{4}$ and $a_{8}$, there is no $B$ satisfying the conditions, that is, there are no cocycles beginning with either $a_{4} e_{36}{ }^{2}$ or $a_{8} e_{36}{ }^{2}$. Recalling that there is no cocycle beginning with either $a_{4} e_{36}$ or $a_{8} e_{38}$, we conclude that $w_{80}=-a_{4}{ }^{2} e_{38}{ }^{2}$ $+\cdots, w_{84}=-a_{4} a_{8} e_{36}{ }^{2}+\cdots$ and $w_{88}=-a_{8}{ }^{2} e_{36}{ }^{2}+\cdots$ are indecomposable.

For $A=a_{20}$ we have $B=-b_{28}{ }^{2}+\left(\partial\right.$-kernel) but no $C$ in $Z_{3}\left[a_{4}, a_{8}, a_{20}, b_{12}\right.$, $\left.b_{18}, b_{28}\right]$ such that $\partial C=B b_{28}=-b_{28}{ }^{3}+$ (other terms). Thus there is no cocycle beginning with $a_{20} e_{36}{ }^{2}$ and we conclude that $w_{96}=-a_{4} a_{20} e_{36}{ }^{2}+\cdots$ and $w_{100}$ $=-a_{8} a_{20} e_{36}{ }^{2}+\cdots$ are indecomposable. However, a cocycle beginning with $a_{20}{ }^{2} e_{36}{ }^{2}$ is decomposable, since $z_{56}{ }^{2}$ begins with $a_{20}{ }^{2} e_{36}{ }^{2}$.

There is no cocycle corresponding to $y_{20}$ as there is no $B$ such that $\partial B$ $=-y_{20} b_{28}$, and there is no cocycle beginning with $y_{20} e_{38}$. Therefore, $v_{96}=a_{4} y_{20} e_{36}{ }^{2}$ $+\cdots, v_{100}=-a_{8} y_{20} e_{36}{ }^{2}+\cdots$ and $u_{112}=-y_{20}{ }^{2} e_{36}{ }^{2}+\cdots$ are indecomposable.

For $A=a_{20} y_{20}$ we have $B=-y_{20} b_{28}{ }^{2}+(\partial$-kernel $)$ but no $C$ such that $\partial C$ $=-y_{20} b_{28}{ }^{3}+$ (other terms). Thus there is no cocycle beginning with $a_{20} y_{20} e_{30}{ }^{2}$. On the other hand, we have a cocycle $v_{112}=\left(a_{20} y_{20}-a_{8} y_{32}\right) e_{30}{ }^{2}+\cdots=\left(-a_{20} y_{20}\right.$ $\left.-a_{4} y_{36}\right) e_{36}{ }^{2}+\cdots$. Hence we conclude here that there is no cocycle beginning with either $a_{8} y_{32} e_{36}{ }^{2}$ or $a_{4} y_{38} e_{36}{ }^{2}$, and also that $v_{112}$ is indecomposable.

For $A=y_{32}$ and $y_{38}$, we have $B=b_{12} b_{28}{ }^{2}+(\partial$-kernel $)$ and $b_{18} b_{28}{ }^{2}+(\partial$-kernel $)$ respectively but no $C$, that is, there is no cocycle corresponding to $y_{32}$ or $y_{38}$. We conclude that $v_{108}, v_{118}, u_{124}$ and $u_{128}$ corresponding respectively to $-a_{4} y_{32}$, $-a_{8} y_{36}, y_{20} y_{32}$ and $-y_{20} y_{36}$ are indecomposable.

One can easily see that cocycles corresponding to $a_{20} y_{32}, a_{20} y_{36}, y_{32}{ }^{2}, y_{36}{ }^{2}$ and $y_{32} y_{36}$ are decomposed in terms of the elements $z_{58}=-a_{20} e_{36}+\cdots, z_{68}=-y_{32}$ $e_{38}+\cdots, z_{68}=-y_{32} e_{38}+\cdots$ and $z_{72}=-y_{36} e_{36}+\cdots$.

We have proved that the cocycles in (iii-2) are all the indecomposable ones of degree 2 with respect to $e_{38}$.

q.e.d.

Obviously we have

Result (iii-3). The element $x_{108}=e_{38}{ }^{3}$ is the only indecomposable cocycle of degree 3 reith respect to $e_{38}$.

It is easy to see that there are no indecomposable cocycles of degree higher than 3. Thus we have shown 
Proposition 3. 10. Cocycles in Results (ii), (iii-1), (iii-2) and (iii-3) are all the indecomposable ones with neither $a_{9}$ nor $c_{17}$. Any cocycle that has neither $a_{9}$ nor $c_{17}$ is trivial if and only if it is 0 as a polynomial in $Z_{3}\left[a_{4}, a_{8}, a_{20}, b_{12}, b_{18}, b_{28}, e_{38}\right]$.

We see

Remark 3.11. (1) The generators are in the $\partial^{2}$-image except $a_{4}, a_{8}$, $y_{20}, x_{36}, x_{48}$ and $x_{108}$;

(2) $a_{4}$ and $a_{8}$ are in the $\partial$-image, but not in the $\partial^{2}$-image;

(3) $y_{20}, x_{38}, x_{48}, x_{108}$ are not in the 0 -image.

Using the above and Lemma 3.8 we see that

(3. 12.1) A cocycle is in the $\partial^{2}$-image if and only if it has no term of the form $a_{4} x_{38}{ }^{i} x_{48}{ }^{j} x_{108}{ }^{k}, a_{8} x_{38}{ }^{i} x_{48}{ }^{j} x_{108}{ }^{k}, x_{38}{ }^{i} x_{48}{ }^{j} x_{108}{ }^{k}$ or $y_{20} x_{38}{ }^{i} x_{48}{ }^{j} x_{108}{ }^{k}$;

(3.12.2) A cocycle is in the D-image but not in the $\partial^{2}$-image if and only if it is a sum of $a_{4} x_{38}{ }^{i} x_{48}{ }^{j} x_{108}{ }^{k}, a_{8} x_{38}{ }^{i} x_{48}{ }^{j} x_{108}{ }^{k}$ and $\partial^{2}$-image;

(3. 12.3) A cocycle is not the $\partial$-image, if it is a sum of $x_{38}{ }^{i} x_{48}{ }^{j} x_{108}{ }^{k}$, $y_{20} x_{38}{ }^{i} x_{48}{ }^{j} x_{108}{ }^{k}$ and any other terms.

\section{$\S$ 4. Elements with $a_{9}$ and $c_{17}$ in $\operatorname{Cotor}_{A}\left(Z_{3}, Z_{3}\right)$}

Now we study cocycles with $a_{9}$ and $c_{17}$.

\section{(iv) Cocycles in $Z_{3}\left\{a_{9}, c_{17}\right\}$}

Clearly $a_{9}$ is a cocycle and $a_{9}{ }^{2}=d c_{17}$. It is easy to see that $x_{26}=\left[a_{9}, c_{17}\right]$ is also a cocycle. The following lemma provides a convenient manner of writing elements in $Z_{3}\left\{a_{9}, c_{17}\right\}$.

Lemma 4. 1. An element $\alpha_{n}$ in $Z_{3}\left\{a_{9}, c_{17}\right\}$ of degree $n$ can be written as follows:

$$
\begin{aligned}
& \alpha_{2 k-1}=d \alpha_{2 k-2}+\sum_{i=0}^{k-1} x_{28}{ }^{i} c_{17} \alpha_{2 k-2 i-2}+\varepsilon x_{28}{ }^{k-1} a_{9}, \\
& \alpha_{2 k}=d \alpha_{2 k-1}+\sum_{i=0}^{k-1} x_{28}{ }^{i} c_{17} \alpha_{2 k-2 i-1}+\varepsilon x_{28}{ }^{k},
\end{aligned}
$$

where $\alpha_{j}$ are elements in $Z_{3}\left\{a_{9}, c_{17}\right\}$ of degree $j$ and $\alpha_{0}, \varepsilon \in Z_{3}$.

Proof. (By induction.) Suppose that the lemma is true for degrees up to $2 k$. Then 


$$
\begin{aligned}
\alpha_{2 k+1}= & \alpha_{2 k} c_{17}+\alpha^{\prime}{ }_{2 k} a_{9} \\
= & \left(d \alpha_{2 k-1}+\sum_{i=0}^{k-1} x_{28}{ }^{i} c_{17} \alpha_{2 k-2 i-1}+\varepsilon x_{26}{ }^{k}\right) c_{17} \\
& +\left(d \alpha^{\prime}{ }_{2 k-1}+\sum_{i=0}^{k-1} x_{28}{ }^{i} c_{17} \alpha^{\prime}{ }_{2 k-2 i-1}+\varepsilon^{\prime} x_{28}{ }^{k}\right) a_{9} \\
= & d\left(\alpha_{2 k-1} c_{17}+\alpha^{\prime}{ }_{2 k-1} a_{9}\right)+\sum_{i=0}^{k-1} x_{28}{ }^{i} c_{17}\left(\alpha_{2 k-2 i-1} c_{17}+\alpha^{\prime}{ }_{2 k-2 i-1} a_{9}\right) \\
& +\varepsilon x_{28}{ }^{k} c_{17}+\varepsilon^{\prime} x_{28}{ }^{k} a_{9}+\alpha_{2 k-1} a_{9}{ }^{2} .
\end{aligned}
$$

Now the last term $\alpha_{2 k-1} a_{9}{ }^{2}$ can be rewritten as follows:

$$
\begin{aligned}
\alpha_{2 k-1} a_{9}{ }^{2} & =\left(d \alpha_{2 k-2}+\sum_{i=0}^{k-1} x_{28}{ }^{i} c_{17} \alpha_{2 k-2 i-2}+\varepsilon^{\prime \prime} x_{28}{ }^{k-1} a_{9}\right) a_{9}{ }^{2} \\
& =d\left(\alpha_{2 k-2} a_{9}{ }^{2}+\varepsilon^{\prime \prime} x_{28}{ }^{k-1} c_{17} a_{9}\right)+\sum_{i=0}^{k-1} x_{28}{ }^{i} c_{17} \alpha_{2 k-2 i-2} a_{9}{ }^{2} .
\end{aligned}
$$

Thus $\alpha_{2 k+1}$ can be written in the required form.

Similarly,

$$
\begin{aligned}
& \alpha_{2 k+2}=\alpha_{2 k+1} c_{17}+\alpha^{\prime}{ }_{2 k+1} a_{9} \\
& =\left(d \alpha_{2 k}+\sum_{i=0}^{k} x_{28}{ }^{i} c_{17} \alpha_{2 k-2 i}+\varepsilon x_{28}{ }^{k} a_{9}\right) c_{17} \\
& +\left(d \alpha^{\prime}{ }_{2 k}+\sum_{i=0}^{k} x_{28}{ }^{i} c_{17} \alpha^{\prime}{ }_{2 k-2 i}+\varepsilon^{\prime} x_{28}{ }^{k} a_{9}\right) a_{9} \\
& =d\left(\alpha_{2 k} c_{17}+\alpha^{\prime}{ }_{2 k} a_{9}+\varepsilon^{\prime} x_{28}{ }^{k} c_{17}\right)+\varepsilon x_{26}{ }^{k+1}-\varepsilon x_{28}{ }^{k} c_{17} a_{9} \\
& +\sum_{i=0}^{k} x_{26}{ }^{i} c_{17}\left(\alpha_{2 k-2 i} c_{17}+\alpha^{\prime}{ }_{2 k-2 i} a_{9}\right)-\alpha_{2 k} a_{9}{ }^{2} \text {, }
\end{aligned}
$$

and the last term $\alpha_{2 k} a_{9}{ }^{2}$ can be rewritten as

$$
\begin{aligned}
\alpha_{2 k} a_{9}{ }^{2} & \left.=d \alpha_{2 k-1}+\sum_{i=0}^{k-1} x_{28}{ }^{i} c_{17} \alpha_{2 k-2 i-1}+\varepsilon^{\prime \prime} x_{28}{ }^{k}\right) a_{9}{ }^{2} \\
& =d\left(\alpha_{2 k-1} a_{9}{ }^{2}+\varepsilon^{\prime \prime} x_{28}{ }^{k} c_{17}\right)+\sum_{i=0}^{k-1} x_{28}{ }^{i} c_{17} \alpha_{2 k-2 i-1} a_{9}{ }^{2} .
\end{aligned}
$$

Hence, $\alpha_{2 k+2}$ can also be written in the required form.

q.e.d.

Proposition 4.2. The elements $a_{9}$ and $x_{28}$ are the only indecomposable cocycles in $\boldsymbol{Z}_{3}\left\{a_{9}, c_{17}\right\}$.

Proof. Writing an element $\alpha_{2 k-1}$ in $Z_{3}\left\{a_{9}, c_{17}\right\}$ of degree $2 k-1$ as in Lemma 4.1, we have

$$
d \alpha_{2 k-1}=\sum_{i=0}^{k-1} x_{28}{ }^{i}\left(a_{9}{ }^{2} \alpha_{2 k-2 i-2}-c_{17} d \alpha_{2 k-2 i-2}\right) .
$$


Thus $d \alpha_{2 k-1}=0$ gives rise to $\alpha_{2 k-2 i-2}=0(0 \leq i \leq k-1)$ (and also $\left.d \alpha_{2 k-2 i-2}=0\right)$. Conversely, if $\alpha_{2 k-2 i-2}=0(0 \leq i \leq k-1)$, then $d \alpha_{2 k-1}$ is clearly 0 . So $\alpha_{2 k-1}$ is a cocycle if and only if it is $\pm x_{28}{ }^{k-1} a_{9}$.

Similarly,

$$
d \alpha_{2 k}=\sum_{i=0}^{k-1} x_{28}{ }^{i}\left(a_{9}{ }^{2} \alpha_{2 k-2 i-1}+c_{17} d \alpha_{2 k-2 i-1}\right)=0
$$

if and only if $\alpha_{2 k-2 i-1}=0(0 \leq i \leq k-1)$ if and only if $\alpha_{2 k}= \pm x_{28}{ }^{k}$.

Therefore $a_{9}$ and $x_{28}$ are the only indecomposable cocycles in $Z_{3}\left\{a_{9}, c_{17}\right\}$.

q.e.d.

\section{(v) Other cocycles}

We shall find other cocycles with $a_{9}$ and $c_{17}$. We shall use the letter $f$ to denote elements in $\bar{W}$.

Lemma 4. 3. An element $f_{n}$ of degree $n$ with respect to $a_{9}$ and $c_{17}$ can be written as

$$
\begin{aligned}
& f_{2 k}=\sum_{i=0}^{k-1} x_{28}{ }^{i} c_{17} f_{2 k-2 i-1}+x_{28}{ }^{k} P+(d \text {-image }), \\
& f_{2 k+1}=\sum_{i=0}^{k-1} x_{28}{ }^{i} c_{17} f_{2 k-2 i}+x_{26}{ }^{k}\left(c_{17} P+a_{9} Q\right)+(d \text {-image }),
\end{aligned}
$$

where $P$ and $Q$ are elements of $R=Z_{3}\left[a_{4}, a_{8}, a_{20}, b_{12}, b_{18}, b_{28}, e_{36}\right]$.

Proof. We shall use the letter $\alpha$ to denote, as before, elements of $\boldsymbol{Z}_{\boldsymbol{s}}$ $\left\{a_{9}, c_{17}\right\}$ and the letter $P$ to denote elements with neither $a_{9}$ nor $c_{17}$. Now, the following identities will be needed in the calculation:

$$
\begin{aligned}
d \alpha_{2 k-1} \cdot P= & d\left(\alpha_{2 k-1} P\right)+\alpha_{2 k-1} d P \\
= & d\left(\alpha_{2 k-1} P\right)+\left(d \alpha_{2 k-2}+\sum_{i=0}^{k-1} x_{28}{ }^{i} c_{17} \alpha_{2 k-2 i-2}+\varepsilon x_{26}{ }^{k-1} a_{9}\right) d P \\
= & d\left(\alpha_{2 k-1} P+\alpha_{2 k-2} d P-\varepsilon x_{26}{ }^{k-1} a_{9} P\right) \\
& +\sum_{i=0}^{k-1} x_{28}{ }^{i} c_{17} \alpha_{2 k-2 i-2}\left(a_{9} \partial P+c_{17} \partial^{2} P\right) \\
= & \sum_{i=0}^{k-1} x_{28}{ }^{i} c_{17} f_{2 k-2 i-1}+(d \text {-image }),
\end{aligned}
$$

and

$$
\begin{aligned}
d \alpha_{2 k} \cdot P & =d\left(\alpha_{2 k} P\right)-\alpha_{2 k} d P \\
& =d\left(\alpha_{2 k} P\right)-\left(d \alpha_{2 k-1}+\sum_{i=0}^{k-1} x_{26}{ }^{i} c_{17} \alpha_{2 k-2 i-1}+\varepsilon x_{26}{ }^{k}\right) d P \\
& =d\left(\alpha_{2 k} P-\alpha_{2 k-1} d P-\varepsilon x_{26}{ }^{k} P\right)-\sum_{i=0}^{k-1} x_{26}{ }^{i} c_{17} \alpha_{2 k-2 i-1}\left(a_{9} \partial P+c_{17} \partial^{2} P\right)
\end{aligned}
$$




$$
=\sum_{i=0}^{k-1} x_{28}{ }^{i} c_{17} f_{2 k-2 i}+(d \text {-image }) \text {. }
$$

Consider now an element of the form $\alpha_{2 k} P$ :

$$
\begin{aligned}
\alpha_{2 k} P & =\left(d \alpha_{2 k-1}+\sum_{i=0}^{k-1} x_{28}{ }^{i} c_{17} \alpha_{2 k-2 i-1}+\varepsilon x_{26}{ }^{k}\right) P \\
& =d \alpha_{2 k-1} \cdot P+\sum_{i=0}^{k-1} x_{26}{ }^{i} c_{17}\left(\alpha_{2 k-2 i-1} P\right)+x_{28}{ }^{k}(\varepsilon P) \\
& =\sum_{i=0}^{k-1} x_{28}{ }^{i} c_{17}\left(f_{2 k-2 i-1}+\alpha_{2 k-2 i-1} P\right)+x_{26}{ }^{k}(\varepsilon P)+(d \text {-image }) .
\end{aligned}
$$

Thus an element $f_{2 k}$ of degree $2 k$ can be written in the required form.

Similarly,

$$
\begin{aligned}
\alpha_{2 k+1} P & =\left(d \alpha_{2 k}+\sum_{i=0}^{k} x_{26}{ }^{i} c_{17} \alpha_{2 k-2 i}+\varepsilon x_{28}{ }^{k} a_{9}\right) P \\
& =\sum_{i=0}^{k-1} x_{28}{ }^{i} c_{17}\left(f_{2 k-2 i}+\alpha_{2 k-2 i} P\right)+x_{26}{ }^{k}\left(c_{17} \alpha_{0} P+\varepsilon a_{9} P\right)
\end{aligned}
$$

and an element $f_{2 k+1}$ can also be written in the required form.

Writing an element of $\bar{W}$ as in the previous lemma, we have

$$
\text { (4. 3. 1) } d f_{2 k}=\sum_{i=0}^{k-1} x_{26}{ }^{i} a_{9}{ }^{2} f_{2 k-2 i-1}-\sum_{i=0}^{k-1} x_{26}{ }^{i} c_{17} d f_{2 k-2 i-1}+x_{26}{ }^{k}\left(a_{9} \partial P+c_{17} \partial^{2} P\right) \text {. }
$$

Thus $d f_{2 k}=0$ gives rise to $f_{2 k-2 i-1}=0 \quad(0 \leq i \leq k-1) \quad$ (and $d f_{2 k-2 i-1}=0$ ) and $\partial P=0$ (that is, $P$ is a cocycle), and the converse is clear. Therefore we have

(4. 4. 1) $d f_{2 k}=0$ if and only if $f_{2 k}$ is of the form $f_{2 k}=x_{26}{ }^{k} A$, where $A$ is a cocycle with neither $a_{9}$ nor $c_{17}$.

Similarly,

(4. 3. 2) $d f_{2 k+1}=\sum_{i=0}^{k-1} x_{28}{ }^{i} a_{9}{ }^{2} f_{2 k-2 i}-\sum_{i=0}^{k-1} x_{28}{ }^{i} c_{17} d f_{2 k-2 i}+x_{28}{ }^{k} a_{9}{ }^{2}(P-\partial Q)$

$$
-x_{28}{ }^{k} c_{17}\left(a_{9} \partial(P-\partial Q)+c_{17} \partial^{2} P\right)-x_{28}{ }^{k+1} \partial^{2} Q \text {. }
$$

Thus $d f_{2 k+1}=0$ if and only if $f_{2 k-2 i}=0 \quad(0 \leq i \leq k-1), P=\partial Q$ and $\partial P$ $=\partial^{2} Q=0$. That is,

(4. 4. 2) $d f_{2 k+1}=0$ if and only if $f_{2 k+1}$ is of the form

$$
f_{2 k+1}=x_{28}{ }^{k}\left(c_{17} \partial Q+a_{9} Q\right) \text { with } \partial^{2} Q=0 .
$$


Thus we have only to determine cocycles of the form

$$
f_{1}=c_{17} \partial Q+a_{9} Q \text { with } \partial^{2} Q=0 .
$$

In case $\partial Q=0, f_{1}$ is a product $a_{9} Q$ with $Q$ a cocycle. We obtain no new cocycle.

In case $\partial Q \neq 0, \partial Q$ is a cocycle as $\partial^{2} Q=0$. If there is another $Q^{\prime}$ such that $\partial Q^{\prime}=\partial Q$, then the difference of $f_{1}=a_{9} Q+c_{17} \partial Q$ and $f_{1}^{\prime}=a_{9} Q^{\prime}+c_{17} \partial Q$ is a decomposable cocycle $a_{9}\left(Q-Q^{\prime}\right)$. Thus, it is sufficient to choose one $Q$ for a cocycle $\partial Q$.

Now, if $\partial Q$ is in the $\partial^{2}$-image, say $\partial Q=\partial^{2} R$, we can choose $Q=\partial R$, but then

$$
f_{1}=a_{9} \partial R+c_{17} \partial^{2} R=d R \text {. }
$$

By (3.12.2), the only cocycle of the form $\partial Q$ but not of the form $\partial^{2} R$ is a sum of

$$
a_{4} x_{38}{ }^{i} x_{48}{ }^{j} x_{108}{ }^{k}, \quad a_{8} x_{38}{ }^{i} x_{48}{ }^{j} x_{108}{ }^{k} \text { and } \partial^{2} \text {-image } \partial^{2} R^{\prime} \text {. }
$$

In particular, for $\partial Q=a_{4}$ and $a_{8}$, taking $Q=-b_{12}$ and $-b_{18}$ respectively, we have

$$
-y_{21}=-a_{9} b_{12}+c_{17} a_{4} \text { and }-y_{25}=-a_{9} b_{16}+c_{17} a_{8} .
$$

For $Q=\sum \alpha(i, j, k) a_{4} x_{38}{ }^{i} x_{48}{ }^{j} x_{108}{ }^{k}+\sum \beta(i, j, k) a_{8} x_{38}{ }^{i} x_{48}{ }^{j} x_{108}{ }^{k}+\partial^{2} R^{\prime} \quad(\alpha(i, j, k)$, $\beta(i, j, k) \in Z_{3}$ and $\left.i, j, k=0,1,2, \cdots\right)$ we have a decomposable cocycle

$$
f_{1}=-\sum \alpha(i, j, k) y_{21} x_{38}{ }^{i} x_{48}{ }^{j} x_{108}{ }^{k}-\sum \beta(i, j, k) y_{25} x_{38}{ }^{i} x_{48}{ }^{j} x_{108}{ }^{k}+d R^{\prime} .
$$

Thus we have

Result (v). We obtain in (v) two new cocycles

$$
y_{21}=a_{9} b_{12}-c_{17} a_{4} \text { and } y_{25}=a_{9} b_{16}-c_{17} a_{8} .
$$

Now we can express (4.4.2) more concretely as follows (incidentally we repeat (4.4.1) just for convenience).

Lemma 4. 4. (1) For an element $f_{2 k}$ of degree $2 k, d f_{2 k}=0$ if and only if $f_{2 k}$ is of the form $x_{26}{ }^{k} A$ with $A$ a cocycle with neither $a_{9}$ nor $c_{17}$.

(2) For an element $f_{2 k+1}$ of degree $2 k+1, d f_{2 k+1}=0$ if and only if $f_{2 k+1}$ is a sum of

$$
x_{28}{ }^{k} a_{9} A, \quad x_{28}{ }^{k} y_{21} x_{38}{ }^{i} x_{48}{ }^{j} x_{108}{ }^{h} \text { and } \quad x_{28}{ }^{k} y_{25} x_{38}{ }^{i} x_{48}{ }^{j} x_{108}{ }^{h} \text {, }
$$

where $A$ is a cocycle with neither $a_{9}$ nor $c_{17}$. 
Now that we have found all generators of $\operatorname{Cotor}_{A}\left(\boldsymbol{Z}_{3}, \boldsymbol{Z}_{3}\right)$, we shall check the commutativity among them and seek relations between generators.

Proposition 4. 5. $\operatorname{Cotor}_{A}\left(\boldsymbol{Z}_{3}, \boldsymbol{Z}_{3}\right)$ is commutative.

Proof. To begin with we have the following $d$-images:

$$
\begin{aligned}
& a_{9}^{2}=d c_{17}, \quad y_{21}{ }^{2}=d\left(c_{17} b_{12}{ }^{2}\right), \quad y_{25}{ }^{2}=d\left(c_{17} b_{18}{ }^{2}\right), \\
& a_{9} y_{21}+x_{28} a_{4}=y_{21} a_{9}-x_{28} a_{4}=-\left[a_{9}, y_{21}\right]=d\left(c_{17} b_{12}\right), \\
& a_{9} y_{25}+x_{26} a_{8}=y_{25} a_{9}-x_{28} a_{8}=-\left[a_{9}, y_{25}\right]=d\left(c_{17} b_{16}\right), \\
& y_{21} y_{25}+x_{26} y_{20}=y_{25} y_{21}-x_{26} y_{20}=-\left[y_{21}, y_{25}\right]=d\left(c_{17} b_{12} b_{16}\right), \\
& {\left[a_{9}, x_{26}\right]=d\left(c_{17}{ }^{2}\right),} \\
& {\left[y_{21}, x_{26}\right]=d\left(c_{17}{ }^{2} b_{12}\right), \quad\left[y_{25}, x_{26}\right]=d\left(c_{17}{ }^{2} b_{18}\right) .}
\end{aligned}
$$

In $\bar{W},\left[a_{9}, P\right]=c_{17} \partial P$ holds. If $A$ is a cocycle with neither $a_{9}$ nor $c_{17}$, we have

(4. 6. 2) $\left[a_{9}, A\right]=0$.

Since $A$ commutes with $a_{9}, c_{17}, a_{4}, a_{8}, b_{12}$ and $b_{16}$, we also have

(4. 6. 3) $\left[y_{21}, A\right]=0,\left[y_{25}, A\right]=0$ and $\left[x_{28}, A\right]=0$.

Therefore commutativity holds in $\operatorname{Cotor}_{A}\left(\boldsymbol{Z}_{3}, \boldsymbol{Z}_{3}\right)$.

q.e.d.

Recall that the differential operator $d$ augments the degree with respect to $a_{9}$ and $c_{17}$ by 1 . Therefore, $\sum f_{l} \in d$-image occurs with different degrees $l$ only when each $f_{l} \in d$-image.

Lemma 4. 7. The following elements are non-trivial:

$$
\begin{array}{ll}
x_{28}{ }^{k} x_{38}{ }^{i} x_{48}{ }^{j} x_{108}{ }^{h}, & x_{28}{ }^{k} y_{20} x_{38}{ }^{i} x_{48}{ }^{j} x_{108}{ }^{h}, \\
x_{28}{ }^{k} a_{4} x_{38}{ }^{i} x_{48}{ }^{j} x_{108}{ }^{h}, & x_{28}{ }^{k} a_{3} x_{38}{ }^{i} x_{48}{ }^{j} x_{108}{ }^{n}, \\
x_{28}{ }^{k} a_{9} x_{38}{ }^{i} x_{48}{ }^{j} x_{108}{ }^{h}, & x_{28}{ }^{k} a_{9} y_{20} x_{38}{ }^{i} x_{48}{ }^{j} x_{108}{ }^{h}, \\
x_{28}{ }^{k} y_{21} x_{38}{ }^{i} x_{48}{ }^{j} x_{108}{ }^{h}, & x_{28}{ }^{k} y_{25} x_{38}{ }^{i} x_{48}{ }^{j} x_{108}{ }^{h}
\end{array}
$$

and they are linearly independent, where $k, i, j, h$ are non-negative integers.

Proof. By Lemma 4.4 a cocycle $f_{2 k+1}$ of degree $2 k+1$ is of the form

$$
\begin{aligned}
f_{2 k+1} & =x_{28}{ }^{k} a_{9} A+x_{26}{ }^{k} \sum \alpha(i, j, h) y_{21} x_{38}{ }^{i} x_{48}{ }^{j} x_{108}{ }^{h}+x_{26}{ }^{k} \sum \beta(i, j, h) y_{25} x_{36}{ }^{i} x_{48}{ }^{j} x_{108}{ }^{h} \\
& =x_{28}{ }^{k}\left(a_{9}\left(A+\sum \alpha(i, j, h) b_{12} x_{38}{ }^{i} x_{48}{ }^{j} x_{108}{ }^{h}+\sum \beta(i, j, h) b_{18} x_{38}{ }^{i} x_{48}{ }^{j} x_{108}{ }^{h}\right)\right.
\end{aligned}
$$




$$
\left.+c_{17}\left(-\sum \alpha(i, j, h) a_{4} x_{38}{ }^{i} x_{48}{ }^{j} x_{108}{ }^{h}-\sum \beta(i, j, h) a_{8} x_{38}{ }^{i} x_{48}{ }^{j} x_{108}{ }^{h}\right)\right) \text {, }
$$

where $\alpha(i, j, h), \beta(i, j, h) \in Z_{3}, A$ is a cocycle with neither $a_{9}$ nor $c_{17}$ and $\sum \alpha(i, j, h) a_{4} x_{38}{ }^{i} x_{48}{ }^{j} x_{108}{ }^{h}+\sum \beta(i, j, h) a_{8} x_{38}{ }^{i} x_{48}{ }^{j} x_{108}{ }^{h}$ is not in the $\partial^{2}$-image by (3. 12.2). On the other hand any element $f_{2 k}$ of degree $2 k$ is written as in Lemma 4. 3:

$$
f_{2 k}=\sum_{i=0}^{k-1} x_{28}{ }^{i} c_{17} f_{2 k-2 i-1}+x_{28}{ }^{k} P+(d \text {-image }),
$$

and its $d$-image is calculated as in (4.3.1):

$$
d f_{2 k}=\sum_{i=0}^{k-1} x_{26}{ }^{i} a_{9}{ }^{2} f_{2 k-2 i-1}-\sum_{i=0}^{k-1} x_{28}{ }^{i} c_{17} d f_{2 k-2 i-1}+x_{26}{ }^{k}\left(a_{9} \partial P+c_{17} \partial^{2} P\right) .
$$

Comparing our cocycle $f_{2 k+1}$ with $d f_{2 k}$, we see that $f_{2 k+1}$ is not in the $d$-image so long as it has a term $x_{28}{ }^{k} y_{21} x_{38}{ }^{i} x_{48}{ }^{j} x_{108}{ }^{n}$ or $x_{28}{ }^{k} y_{25} x_{38}{ }^{i} x_{48}{ }^{j} x_{108}{ }^{h}$. That is to say, $x_{26}{ }^{k} y_{21} x_{38}{ }^{i} x_{48}{ }^{j} x_{108}{ }^{h}$ and $x_{28}{ }^{k} y_{25} x_{36}{ }^{i} x_{48}{ }^{j} x_{108}{ }^{h}$ are non-trivial, and they and $x_{28}{ }^{k} a_{9} A$ (if the latter is non-trivial) are linearly independent.

Comparing $x_{28}{ }^{i} a_{9} A$ again with $d f_{2 k}$, we see that $x_{28}{ }^{k} a_{9} A$ is in the $d$-image only when $A=\partial P$ and $x_{28}{ }^{k} a_{9} \partial P=d\left(x_{28}{ }^{k} P\right)$. Referring to (3.12.3), we see that

$$
x_{28}{ }^{k} a_{9} x_{36}{ }^{i} x_{48}{ }^{j} x_{108}{ }^{h}, \quad x_{28}{ }^{k} a_{9} y_{20} x_{38}{ }^{i} x_{48}{ }^{j} x_{108}{ }^{h}
$$

and their sum are non-trivial.

A cocycle of degree $2 k+2$ is, by Lemma 4. 4, of the form $x_{26}{ }^{k+1} A$ where $A$ is a cocycle with neither $a_{9}$ nor $c_{17}$. And any element $f_{2 k+1}$ of degree $2 k$ +1 is written as in Lemma 4.3 :

$$
f_{2 k+1}=\sum_{i=0}^{k-1} x_{26}{ }^{i} c_{17} f_{2 k-2 i}+x_{26}{ }^{k}\left(c_{17} P+a_{9} Q\right)+(d \text {-image }),
$$

and its $d$-image is calculated as (4.3.2):

$$
\begin{aligned}
d f_{2 k+1}= & \sum_{i=0}^{k-1} x_{28}{ }^{i} a_{9}{ }^{2} f_{2 k-2 i}-\sum_{i=0}^{k-1} x_{28}{ }^{i} c_{17} d f_{2 k-2 i}+x_{28}{ }^{k} a_{9}{ }^{2}(P-\partial Q) \\
& -x_{28}{ }^{k} c_{17}\left(a_{9} \partial(P-\partial Q)+c_{17} \partial^{2} P\right)-x_{28}{ }^{k+1} \partial^{2} Q .
\end{aligned}
$$

Comparing $x_{28}{ }^{k+1} A$ with $d f_{2 k+1}$, we see that $x_{26}{ }^{k+1} A$ is in the $d$-image only when $A=\partial^{2}(-Q)$, and then

$$
x_{28}{ }^{k+1} \partial^{2}(-Q)=d\left(x_{26}{ }^{k}\left(a_{9} Q+c_{17} \partial Q\right)\right) .
$$

So by (3.12.2) and (3.12.3) we see that

$$
x_{26}{ }^{k} x_{38}{ }^{i} x_{48}{ }^{j} x_{108}{ }^{h}, \quad x_{28}{ }^{k} y_{20} x_{38}{ }^{i} x_{48}{ }^{j} x_{108}{ }^{h},
$$




$$
x_{28}{ }^{k} a_{4} x_{38}{ }^{i} x_{48}{ }^{j} x_{108}{ }^{h}, \quad x_{28}{ }^{k} a_{8} x_{38}{ }^{i} x_{48}{ }^{j} x_{108}{ }^{n}
$$

and their sum are non-trivial.

q.e.d.

We prove

Lemma 4. 8. A cocycle with $a_{9}$ and $c_{17}$ is either trivial or a linear combination of cocyles in Lemma 4.7 .

Proof. Recall from (4.6.1) the relations:

$$
\begin{array}{ll}
a_{9}{ }^{2}=d c_{17}, \quad y_{21}{ }^{2}=d\left(c_{17} b_{12}{ }^{2}\right), & y_{25}{ }^{2}=d\left(c_{17} b_{16}{ }^{2}\right), \\
a_{9} y_{21}=-x_{26} a_{4}+d\left(c_{17} b_{12}\right), & a_{9} y_{25}=-x_{26} a_{8}+d\left(c_{17} b_{16}\right), \\
y_{21} y_{25}=-x_{26} y_{20}+d\left(c_{17} b_{12} b_{16}\right) . &
\end{array}
$$

Thus any cocycle is reduced to a cocycle, each term of which has at most one of $a_{9}, y_{21}$ or $y_{25}$.

We have shown that

(4. 9. 1) $x_{28} \partial^{2} Q=d\left(-a_{9} Q-c_{17} \partial Q\right)$,

and $a_{9} \partial P=d P$, in pariticular,

(4. 9. 2) $\quad a_{9} \partial^{2} Q=d(\partial Q), \quad a_{9} a_{4}=d\left(-b_{12}\right), \quad a_{9} a_{8}=d\left(-b_{16}\right)$.

Finally, we have the following $d$-images:

$$
\begin{array}{ll}
y_{21} \partial^{2} Q=d\left(a_{4} Q+b_{12} \partial Q\right), & y_{25} \partial^{2} Q=d\left(a_{8} Q+b_{18} \partial Q\right), \\
y_{21} a_{4}=d\left(b_{12}{ }^{2}\right), & y_{25} a_{8}=d\left(b_{16}{ }^{2}\right), \\
y_{21} a_{8}+a_{9} y_{20}=y_{25} a_{4}-a_{9} y_{20}=d\left(b_{12} b_{16}\right), & \\
y_{21} y_{20}=d\left(-b_{12}{ }^{2} b_{16}\right), & y_{25} y_{20}=d\left(b_{12} b_{16}{ }^{2}\right) .
\end{array}
$$

Using these relations we see that any monomial in cocycles is either trivial or equivalent to one of cocycles in Lemma 4.7.

q.e.d.

Theorem 4. 10. For $A=H^{*}\left(X_{7} ; Z_{3}\right)$, we have as algebra

$\operatorname{Cotor}_{A}\left(\boldsymbol{Z}_{3}, \boldsymbol{Z}_{3}\right) \cong \boldsymbol{Z}_{3}\left[a_{9}, y_{21}, y_{25}, x_{28}, a_{4}, a_{8}, a_{20}, x_{38}, x_{48}, x_{84}, x_{108}\right.$, $y_{20}, y_{32}, y_{36}, y_{68}, y_{80}, y_{84}, y_{96}, z_{56}, z_{44}, z_{48}, z_{52}, z_{88}, z_{72}, z_{80}$, $z_{64}, z_{76}, z_{104}, z_{116}, z_{120}, z_{132}, w_{80}, w_{84}, w_{88}, w_{96}, w_{100}$, $\left.v_{98}, v_{100}, v_{108}, v_{112}, v_{116}, u_{112}, u_{124}, u_{128}, p_{140}, p_{152}, p_{156}, p_{188}\right] / \rho$,

where $\rho$ is the ideal generated by

i) elements which are 0 as polynomial in $Z_{3}\left[a_{4}, a_{8}, a_{20}, b_{12}, b_{18}, b_{28}, e_{38}\right]$,

ii) $a_{9}{ }^{2}, y_{21}{ }^{2}, y_{25}{ }^{2}$, 
iii) $a_{9} \partial^{2} Q, \quad y_{21} \partial^{2} Q, \quad y_{25} \partial^{2} Q, \quad x_{28} \partial^{2} Q$,

$a_{9} a_{4}, \quad a_{9} a_{8}, \quad y_{21} a_{4}, \quad y_{25} a_{8}, \quad y_{21} y_{20}, \quad y_{25} y_{20}$.

$y_{21} a_{8}+a_{9} y_{20}=y_{25} a_{4}-a_{9} y_{20}=y_{21} a_{8}+y_{25} a_{4}$.

(See Results (ii), (iii-1), (iii-2), (iii-3) and (v) for the expression of the generators in terms of $a_{i}{ }^{\prime} s, b_{j}{ }^{\prime} s, c_{17}$ and $e_{36}$. See also Remark 3.11 for practical use of the $\partial^{2}$-image.)

\section{§ 5. $\operatorname{Cotor}_{B}\left(Z_{3}, Z_{3}\right)$ with $B=H^{*}\left(X_{6} ; Z_{3}\right)$}

The Hopf algebra structure of $H^{*}\left(X_{\theta} ; Z_{3}\right)$ is very alike that of $H^{*}\left(X_{7}\right.$; $\left.Z_{3}\right)([6]$ and $[7])$ :

$$
H^{*}\left(X_{6} ; Z_{3}\right) \cong Z_{3}\left[x_{8}\right] /\left(x_{8}^{3}\right) \otimes \Lambda\left(x_{3}, x_{7}, x_{9}, x_{11}, x_{15}, x_{17}\right),
$$

where $\operatorname{deg} x_{i}=i$;

$$
\begin{array}{ll}
\bar{\phi}\left(x_{i}\right)=0 & \text { for } i=3,7,8,9, \\
\bar{\phi}\left(x_{j}\right)=x_{8} \otimes x_{j-8} & \text { for } j=11,15,17,
\end{array}
$$

where $\bar{\phi}$ is the reduced diagonal map induced from the multiplication on $X_{6}$.

Notation. $B=H^{*}\left(\mathrm{X}_{6} ; Z_{3}\right)$ and $\bar{B}=\widetilde{H}^{*}\left(X_{6} ; Z_{3}\right)$.

We construct an injective resolution of $Z_{3}$ over $B$ quite similarly to that in $\S 2$, taking $M$ to be a graded $Z_{3}$-submodule of $\bar{B}$ generated by

$$
\left\{x_{3}, x_{7}, x_{8}, x_{9}, x_{11}, x_{15}, x_{17}, x_{8}^{2}\right\}
$$

and naming the set of the corresponding elements under the suspension $s$ as

$$
s M=\left\{a_{4}, a_{8}, a_{9}, a_{10}, b_{12}, b_{18}, b_{18}, c_{17}\right\} .
$$

Now, we put

$$
\begin{aligned}
\bar{V} & =T(s M) / J \\
& =Z_{3}\left\{a_{4}, a_{8}, a_{9}, a_{10}, b_{12}, b_{18}, b_{18}, c_{17}\right\} / J,
\end{aligned}
$$

where $J$ is the ideal generated by

(5. 4) $[\alpha, \beta]$ for all pairs $(\alpha, \beta)$ of generators of $\bar{V}$ except $\left(a_{9}, b_{j}\right)$ $(j=12,16,18)$ and $\left(a_{9}, c_{17}\right)$,

$$
\left[a_{9}, b_{j}\right]+c_{17} a_{j-8} \quad \text { for } j=12,16,18,
$$

where $[\alpha, \beta]=\alpha \beta-(-1) * \beta \alpha$ with $*=\operatorname{deg} \alpha \cdot \operatorname{deg} \beta$. 
We construct the twisted tensor product $V=B \otimes \bar{V}$ as in $\S 2$. Then the differential operators $\bar{d}$ in $V$ and $d$ in $\bar{V}$ are given by

$$
\begin{array}{lr}
\bar{d}\left(x_{i} \otimes 1\right)=1 \otimes a_{i+1} & \text { for } i=3,7,8,9, \\
\bar{d}\left(x_{8}{ }^{2} \otimes 1\right)=1 \otimes c_{17}-x_{8} \otimes a_{9} & \\
\bar{d}\left(x_{j} \otimes 1\right)=1 \otimes b_{j+1}+x_{8} \otimes a_{j-7} & \text { for } j=11,15,17 ; \\
d a_{i}=0 & \text { for } i=3,8,9,10, \\
d c_{17}=a_{9}{ }^{2}, & \\
d b_{j}=-a_{9} a_{j-8} & \text { for } j=12,16,18 .
\end{array}
$$

Note that $\bar{V}$ contains the polynomial algebra $S=Z_{3}\left[a_{4}, a_{8}, a_{10}, b_{12}, b_{16}, b_{18}\right]$. Quite similarly as before one can show

Theorem 5. 7. $V$ is an injective resolution of $\boldsymbol{Z}_{3}$ over $B=H^{*}\left(X_{8} ; \boldsymbol{Z}_{3}\right)$.

Corollary 5. 8. $H(\bar{V}: d)=\operatorname{Ker} d / \operatorname{Im} d \cong \operatorname{Cotor}_{B}\left(\boldsymbol{Z}_{3}, \boldsymbol{Z}_{3}\right)$.

Before we calculate $H(\bar{V}: d)$ we observe that the Hopf algebra $H^{*}\left(X_{6}\right.$; $\left.Z_{3}\right)$ is obtained by replacing $x_{19}$ and $x_{27}$ in $H^{*}\left(X_{7} ; Z_{3}\right)$ with $x_{9}$ and $x_{17}$ respectively and by omitting $x_{35}$ in $H^{*}\left(X_{7} ; Z_{3}\right)$. This corresponds to the fact that $\bar{V}$ is obtained by replacing $a_{20}$ and $b_{28}$ in $\bar{W}$ with $a_{10}$ and $b_{18}$ respectively and by omitting $e_{36}$ in $\bar{W}$. Thus the calculation of cocycles is done almost similarly to but more simply than the case of $X_{7}$.

Parallel to the case of $X_{7}$ we shall find cocycles in the following steps:

(i)' cocycles in $Z_{3}\left[a_{4}, a_{8}, a_{10}, b_{12}, b_{18}\right]$,

(ii)' those in $Z_{3}\left[a_{4}, a_{8}, a_{10}, b_{12}, b_{18}, b_{18}\right]$,

(iii)' (this is not necessary, since there is no $e_{36}$ ),

(iv)' those in $Z_{3}\left\{a_{9}, c_{17}\right\}$,

(v)' other cocycles.

In order to calculate $H(\bar{V}: d)$ we define an operator which we denote also by $\partial$ :

$$
\begin{array}{lll}
\partial a_{4}=0, & \partial a_{8}=0, & \partial a_{10}=0, \\
\partial b_{12}=-a_{4}, & \partial b_{18}=-a_{8}, & \partial b_{18}=-a_{10},
\end{array}
$$

and extend it over $S=Z_{3}\left[a_{4}, a_{8}, a_{10}, b_{12}, b_{18}, b_{18}\right]$ by (3.2). Lemmas 3.3 and 3.4 again hold for $P$ in $S$.

\section{(i) ', (ii)', (iii)' Cocycles in $Z_{3}\left[a_{4}, a_{8}, a_{10}, b_{12}, b_{16}, b_{18}\right]$}

The calculation of cocycles with neither $a_{9}$ nor $c_{17}$ is as above except that we have no step (iii): 
Proposition 5. 10. We have the following indecomposable cocycles with neither $a_{9}$ nor $c_{17}$ :

$$
\begin{aligned}
& a_{4}, a_{8}, a_{10}, \\
& x_{36}=b_{12}^{3}, x_{48}=b_{18}{ }^{3}, x_{54}=b_{18}^{3}, \\
& y_{20}=a_{8} b_{12}-a_{4} b_{18}, y_{22}=a_{4} b_{18}-a_{10} b_{12}, y_{28}=a_{8} b_{18}-a_{10} b_{16}, \\
& y_{58}=\partial^{2}\left(b_{12}{ }^{2} b_{18}{ }^{2} b_{18}\right), y_{60}=\partial^{2}\left(b_{12}{ }^{2} b_{18} b_{18}{ }^{2}\right), \\
& y_{64}=\partial^{2}\left(b_{12} b_{18}^{2} b_{18}{ }^{2}\right), y_{76}=\partial^{2}\left(b_{12}{ }^{2} b_{18}{ }^{2} b_{18}^{2}\right) .
\end{aligned}
$$

Lemma 5. 11. The elements $y_{58}, y_{60}, y_{64}, y_{78}$ and the following products appear in the $\partial^{2}$-image:

$$
\begin{array}{lll}
a_{4}{ }^{2}=\partial^{2}\left(-b_{12}{ }^{2}\right), & a_{8}{ }^{2}=\partial^{2}\left(-b_{18}{ }^{2}\right), & a_{10}{ }^{2}=\partial^{2}\left(-b_{18}{ }^{2}\right), \\
a_{4} a_{8}=\partial^{2}\left(-b_{12} b_{18}\right), & a_{4} a_{10}=\partial^{2}\left(-b_{12} b_{18}\right), & a_{8} a_{10}=\partial^{2}\left(-b_{18} b_{18}\right), \\
a_{4} y_{20}=\partial^{2}\left(b_{12}{ }^{2} b_{18}\right), & a_{8} y_{20}=\partial^{2}\left(-b_{12} b_{18}{ }^{2}\right), & \\
a_{4} y_{22}=\partial^{2}\left(-b_{12}{ }^{2} b_{18}\right), & a_{10} y_{22}=\partial^{2}\left(b_{12} b_{18}{ }^{2}\right), \\
a_{8} y_{28}=\partial^{2}\left(-b_{18}{ }^{2} b_{18}\right), & a_{10} y_{28}=\partial^{2}\left(b_{18} b_{18}{ }^{2}\right), \\
a_{4} y_{28}+a_{8} y_{22}=-a_{8} y_{22}+a_{10} y_{20}=-a_{10} y_{20}-a_{4} y_{28}=\partial^{2}\left(b_{12} b_{18} b_{18}\right), \\
y_{20}{ }^{2}=\partial^{2}\left(-b_{12}{ }^{2} b_{18}{ }^{2}\right), & y_{22}{ }^{2}=\partial^{2}\left(-b_{12}{ }^{2} b_{18}{ }^{2}\right), & y_{26}{ }^{2}=\partial^{2}\left(-b_{18}{ }^{2} b_{18}{ }^{2}\right), \\
y_{20} y_{22}=\partial^{2}\left(b_{12}{ }^{2} b_{18} b_{18}\right), & y_{20} y_{28}=\partial^{2}\left(-b_{12} b_{18}{ }^{2} b_{18}\right), y_{22} y_{28}=\partial^{2}\left(-b_{12} b_{18} b_{18}{ }^{2}\right),
\end{array}
$$

and $P \cdot \partial^{2} Q=\partial^{2}(P Q)$ for any cocycle $P$ with neither $a_{9}$ nor $c_{17}$.

Lemma 5.11 is just the interpretation of the corresponding Lemma 3. 8. Note the following:

(5.11.1) The generators $a_{10}, y_{22}, y_{26}$ and $x_{54}$ correspond to $a_{20}=\partial^{2} e_{38}, y_{32}$ $=\partial^{2}\left(-b_{12} e_{38}\right), y_{38}=\partial^{2}\left(-b_{18} e_{38}\right)$ and $x_{84}=\partial^{2}\left(-b_{28} e_{38}{ }^{2}\right)$ respectively in the case of $X_{7}$, but they are not in the $\partial^{2}$-image;

(5. 11.2) In Lemma 5. 11 we have a $\partial^{2}$-image

$$
a_{10} y_{20}-a_{8} y_{22}=-a_{10} y_{20}-a_{4} y_{28}=a_{4} y_{28}+a_{8} y_{22}=\partial^{2}\left(b_{12} b_{18} b_{18}\right),
$$

though $a_{10} y_{20}, a_{8} y_{22}$ and $a_{4} y_{28}$ are not in the $\partial^{2}$-image, which did not occur in the final résumé of $\partial^{2}$-image in $X_{7}$ (Remark (3.5)).

Remark 5. 12. Of the generators,

(1) $y_{58}, y_{60}, y_{64}$ and $y_{78}$ are in the $\partial^{2}$-image; 
(2) $a_{4}=\partial\left(-b_{12}\right), a_{8}=\partial\left(-b_{18}\right)$ and $a_{10}=\partial\left(-b_{18}\right)$ are in the $\partial$-image, but not in the $\partial^{2}$-image;

(3) $y_{20}, y_{22}, y_{28}, x_{36}, x_{48}$ and $x_{54}$ are not in the $\partial$-image.

Using the above and Lemma 5.11 we see that

(5. 12.1) Monomials in cocycles reith neither $a_{9}$ nor $c_{17}$ except the ones in (5.12.2) and (5.12.3) are in the $\partial^{2}$-image;

(5. 12.2)

$$
\begin{aligned}
& a_{4} x_{38}{ }^{i} x_{48}{ }^{j} x_{54}{ }^{k}, a_{8} x_{36}{ }^{i} x_{48}{ }^{j} x_{54}{ }^{k}, a_{10} x_{38}{ }^{i} x_{48}{ }^{j} x_{54}{ }^{k}, \\
& a_{4} y_{28} x_{38}{ }^{i} x_{48}{ }^{j} x_{54}{ }^{k}, a_{8} y_{22} x_{38}{ }^{i} x_{48}{ }^{j} x_{54}{ }^{k} \text { and } a_{10} y_{20} x_{38}{ }^{i} x_{48}{ }^{j} x_{54}{ }^{k}
\end{aligned}
$$

are in the 0 -image, but not in the $\partial^{2}$-image:

(5. 12. 3) $x_{36}{ }^{i} x_{48}{ }^{j} x_{54}{ }^{k}, y_{20} x_{38}{ }^{i} x_{48}{ }^{j} x_{54}{ }^{k}, y_{22} x_{98}{ }^{i} x_{48}{ }^{j} x_{54}{ }^{k}$ and $y_{28} x_{38}{ }^{i} x_{48}{ }^{j} x_{54}{ }^{k}$ are not in the D-image.

Note (cf. (5.11.2)) that

$$
\begin{aligned}
& a_{4} y_{28} x_{38}{ }^{i} x_{48}{ }^{j} x_{54}{ }^{k}=-a_{10} y_{20} x_{38}{ }^{i} x_{48}{ }^{j} x_{54}{ }^{k}+\left(\partial^{2} \text {-image }\right), \\
& a_{8} y_{22} \cdot x_{36}{ }^{i} x_{48}{ }^{j} x_{54}{ }^{k}=a_{10} y_{20} x_{38}{ }^{i} x_{48}{ }^{j} x_{54}{ }^{k}+\left(\partial^{2} \text {-image }\right) .
\end{aligned}
$$

From now on until the end of the calculation of $\operatorname{Cotor}_{B}\left(\boldsymbol{Z}_{3}, \boldsymbol{Z}_{3}\right)$, we shall always replace $a_{4} y_{28} x_{38}{ }^{i} x_{48}{ }^{j} x_{54}{ }^{k}$ and $a_{8} y_{22} x_{38}{ }^{i} x_{48}{ }^{j} x_{54}{ }^{k}$ by the right hand sides of the above relations.

Such replacement done, we have the following:

(5. 13.1) A cocycle is in the $\partial^{2}$-image if and only if each term of the cocycle is in the $\partial^{2}$-image;

(5. 13.2) A cocylce is in the $\partial$-image but not in the $\partial^{2}$-image if and only if it is of the form

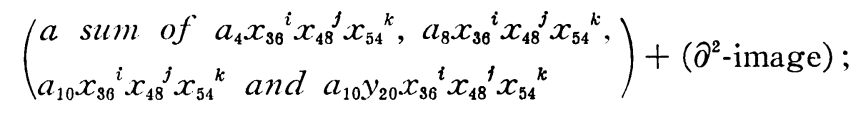

(5. 13. 3) A cocycle is not in the $\partial$-image if and only if it is of the form (a sum of monomials in (5.12.3)) +(any cocycle).

\section{(iv)' Cocycles in $Z_{3}\left\{a_{9}, c_{17}\right\}$}

No change is needed in step (iv)' and we obtain

Proposition 5. 13. The elements $a_{9}$ and $x_{20}=\left[a_{9}, c_{17}\right]$ are the only indecomposable cocycles in $Z_{3}\left\{a_{9}, c_{17}\right\}$. 


\section{$(\mathbf{v})^{\prime}$ Other cocycles}

The argument is almost the same as in (v) and we have

(5. 14. 1) For an element $f_{2 k}$ of degree $2 k$ with respect to $a_{9}$ and $c_{17}$, $d f_{2 k}=0$ if and only if $f_{2 k}$ is of the form

$$
f_{2 k}=x_{26}{ }^{k} A \text { with } A \text { a cocycle with neither } a_{9} \text { nor } c_{17} \text {; }
$$

(5. 14. 2) For an element $f_{2 k+1}$ of degree $2 k+1$ with respect to $a_{9}$ and $c_{17}$. $d f_{2 k+1}=0$ if and only if $f_{2 k+1}$ is of the form

$$
f_{2 k+1}=x_{28}{ }^{k}\left(a_{9} Q+c_{17} \partial Q\right) \quad \text { with } \partial^{2} Q=0 .
$$

Using these formulae we see that we have only to determine cocycles of the form

$$
f_{1}=a_{9} Q+c_{17} \partial Q \quad \text { with } \partial^{2} Q=0 .
$$

In case $\partial Q=0, f_{1}$ is a decomposable cocycle $a_{9} Q$ with $Q$ a cocycle.

In case $\partial Q \neq 0, \partial Q$ is a cocycle as $\partial^{2} Q=0$. If $\partial Q=\partial^{2} R$ for some $R$, then choosing $Q$ to be $\partial R$, we have $f_{1}=a_{9} \partial R+c_{17} \partial^{2} R=d R$, which is a trivial cocycle. By (5.13.2) a cocycle of the form $\partial Q$ but not of the form $\partial^{2} R$ is a sum of

$$
a_{4} x_{36}{ }^{i} x_{48}{ }^{j} x_{54}{ }^{k}, a_{8} x_{38}{ }^{i} x_{48}{ }^{j} x_{54}{ }^{k}, a_{10} x_{36}{ }^{i} x_{48}{ }^{j} x_{54}{ }^{k}, a_{10} y_{20} x_{38}{ }^{i} x_{48}{ }^{j} x_{54}{ }^{k} \text { and ( } \partial^{2} \text {-image) } \text {. }
$$

In particular, for $a_{4}=\partial\left(-b_{12}\right), a_{8}=\partial\left(-b_{18}\right)$ and $a_{10}=\partial\left(-b_{18}\right)$, we have

$$
y_{21}=a_{9} b_{12}-c_{17} a_{4}, y_{25}=a_{9} b_{16}-c_{17} a_{8} \text { and } y_{27}=a_{9} b_{18}-c_{17} a_{10} .
$$

And for $\partial Q$ of the form of a sum above, we have a sum of

$$
\begin{aligned}
& -y_{21} x_{38}{ }^{i} x_{48}{ }^{j} x_{54}{ }^{k},-y_{25} x_{38}{ }^{i} x_{48}{ }^{j} x_{54}{ }^{k}, \\
& -y_{27} a_{10} x_{38}{ }^{i} x_{48}{ }^{j} x_{54}{ }^{k},-y_{27} y_{20} x_{38}{ }^{i} x_{48}{ }^{j} x_{54}{ }^{k} \text { and (d-image). }
\end{aligned}
$$

Thus we have three new indecomposable cocycles with $a_{9}$ and $c_{17}$, namely, $y_{21}, y_{25}$ and $y_{27}$.

Remark. The cocycles $y_{21}$ and $y_{25}$ are the same as in (v), and the cocycle in (v) that corresponds to $y_{27}$ is a trivial one $a_{9} b_{28}-c_{17} a_{20}=d\left(-e_{38}\right)$.

Looking at $(5.12 .1) \sim(5.12 .3)$, we see that we have found all cocycles to be found in (v)'.

Result (v)'. We have three cocycles in step (v)':

$$
y_{21}=a_{9} b_{12}-c_{17} a_{4}, y_{25}=a_{9} b_{18}-c_{17} a_{8} \text { and } y_{27}=a_{9} b_{18}-c_{17} a_{10} \text {. }
$$


We have seen also

Lemma 5. 14. (1) For an element $f_{2 k}$ of degree $2 k, d f_{2 k}=0$ if and only if $f_{2 k}$ is of the form $x_{26}{ }^{k} A$ where $A$ is a cocycle with neither $a_{9}$ nor $c_{17}$ :

(2) For an element $f_{2 k+1}$ of degree $2 k+1, d f_{2 k+1}=0$ if and only if $f_{2 k+1}$ is of the form

$$
x_{26}{ }^{k}\left\{a_{9} A+\left(\begin{array}{cccc}
a & \text { sum } & \text { of } y_{21} x_{38}{ }^{i} x_{48}{ }^{j} x_{54}{ }^{h}, y_{25} x_{38}{ }^{i} x_{48}{ }^{j} x_{54}{ }^{h} \\
y_{27} x_{38}{ }^{i} x_{48}{ }^{j} x_{54}{ }^{h} \text { and } y_{27} y_{20} x_{38}{ }^{i} x_{48}{ }^{j} x_{54}{ }^{h},
\end{array}\right)\right\}
$$

where $A$ is a cocycle with neither $a_{9}$ nor $c_{17}$.

Here we have the following $d$-images:

(5. 15. 1) In addition to the d-images in (4.6.1) we have

$$
\begin{aligned}
& y_{27}{ }^{2}=d\left(c_{17} b_{18}{ }^{2}\right),\left[y_{27}, x_{28}\right]=d\left(c_{17}{ }^{2} b_{18}\right), \\
& a_{9} y_{27}+x_{28} a_{10}=y_{27} a_{9}-x_{28} a_{10}=-\left[a_{9}, y_{27}\right]=d\left(c_{17} b_{18}\right), \\
& y_{21} y_{27}-x_{28} y_{22}=y_{27} y_{21}+x_{28} y_{22}=-\left[y_{21}, y_{27}\right]=d\left(c_{17} b_{12} b_{18}\right), \\
& y_{25} y_{27}-x_{26} y_{28}=y_{27} y_{25}+x_{26} y_{26}=-\left[y_{25}, y_{27}\right]=d\left(c_{17} b_{12} b_{18}\right) .
\end{aligned}
$$

In addition to the relations (4.6.2) and (4.6.3) we have similarly

(5. 15. 2) $\left[y_{27}, P\right]=0$ for $P$ with neither $a_{9}$ nor $c_{17}$.

Thus we have

Proposition 5. 16. $\operatorname{Cotor}_{B}\left(\boldsymbol{Z}_{3}, \boldsymbol{Z}_{3}\right)$ is commutative.

Lemma 5. 17. The following elements are non-trivial and they are linearly independent.

$$
\begin{aligned}
& x_{28}{ }^{k} x_{36}{ }^{i} x_{48}{ }^{j} x_{54}{ }^{h}, \quad x_{28}{ }^{k} a_{4} x_{38}{ }^{i} x_{48}{ }^{j} x_{54}{ }^{h}, \quad x_{28}{ }^{k} a_{8} x_{38}{ }^{i} x_{48}{ }^{j} x_{54}{ }^{h}, \quad x_{28}{ }^{k} a_{10} x_{38}{ }^{i} x_{48}{ }^{j} x_{54}{ }^{h}, \\
& x_{28}{ }^{k} y_{20} x_{88}{ }^{i} x_{48}{ }^{j} x_{54}{ }^{h}, x_{28}{ }^{k} y_{22} x_{38}{ }^{i} x_{48}{ }^{j} x_{54}{ }^{h}, \quad x_{28}{ }^{k} y_{28} x_{38}{ }^{i} x_{48}{ }^{j} x_{54}{ }^{h}, \quad x_{28}{ }^{k} a_{10} y_{20} x_{38}{ }^{i} x_{48}{ }^{j} x_{54}{ }^{h} \text {, } \\
& x_{28}{ }^{k} a_{9} \cdot x_{38}{ }^{i} x_{48}{ }^{j} x_{54}{ }^{h}, \quad x_{28}{ }^{k} a_{9} y_{20} x_{38}{ }^{i} x_{48}{ }^{j} x_{54}{ }^{h}, \quad x_{28}{ }^{k} a_{9} y_{22} x_{38}{ }^{i} x_{48}{ }^{j} x_{54}{ }^{h}, \quad x_{28}{ }^{k} a_{9} y_{28} x_{38}{ }^{i} x_{48}{ }^{j} x_{54}{ }^{h} \text {, } \\
& x_{26}{ }^{k} y_{21} x_{36}{ }^{i} x_{48}{ }^{j} x_{54}{ }^{h}, \quad x_{28}{ }^{k} y_{25} x_{38}{ }^{i} x_{48}{ }^{j} x_{54}{ }^{h}, \quad x_{28}{ }^{k} y_{27} x_{38}{ }^{i} x_{48}{ }^{j} x_{54}{ }^{h}, \quad x_{26}{ }^{k} y_{27} y_{20} x_{38}{ }^{i} x_{48}{ }^{j} x_{54}{ }^{h} \text {. }
\end{aligned}
$$

Proof. The argument is the same as in the proof of Lemma 4.7.

A cocycle $f_{2 k+1}$ of degree $2 k+1$ is, by Lemma 5.14 , of the form

$$
\begin{aligned}
x_{28}{ }^{k} a_{9} A & +\left(\begin{array}{l}
\text { a sum of } x_{28}{ }^{k} y_{21} x_{38}{ }^{i} x_{48}{ }^{j} x_{54}{ }^{h}, x_{28}{ }^{k} y_{25} x_{38}{ }^{i} x_{48}{ }^{j} x_{54}{ }^{h}, \\
x_{28}{ }^{k} y_{27} x_{38}{ }^{i} x_{48}{ }^{j} x_{54}{ }^{h} \text { and } x_{28}{ }^{k} y_{27} y_{20} x_{38}{ }^{i} x_{48}{ }^{j} x_{54}{ }^{h}
\end{array}\right) \\
& =x_{28}{ }^{k}\left(a_{9} Q+c_{17} \partial Q\right),
\end{aligned}
$$


where the $\partial Q$ was taken not to be in the $\partial^{2}$-image and $A$ is a cocycle with neither $a_{9}$ nor $c_{17}$. Comparing $f_{2 k+1}$ with $d f_{2 k}$ as in the proof of Lemma 4.7, we see that such an $f_{2 k+1}$ is not in the $d$-image so long as it has a term $x_{28}{ }^{k} y_{21} x_{38}{ }^{i} x_{48}{ }^{j} x_{54}{ }^{h}, \quad x_{28}{ }^{k} y_{25} x_{38}{ }^{i} x_{48}{ }^{j} x_{54}{ }^{h}, \quad x_{28}{ }^{k} y_{27} x_{38}{ }^{i} x_{48}{ }^{j} x_{54}{ }^{h}$ or $x_{26}{ }^{k} y_{27} y_{20} x_{38}{ }^{i} x_{48}{ }^{j} x_{54}{ }^{h}$. In other words, $x_{28}{ }^{k} y_{21} x_{38}{ }^{i} x_{48}{ }^{j} x_{54}{ }^{h}, x_{28}{ }^{k} y_{25} x_{38}{ }^{i} x_{48}{ }^{j} x_{54}{ }^{h}, x_{28}{ }^{k} y_{27} x_{36}{ }^{i} x_{48}{ }^{j} x_{54}{ }^{h}$ and $x_{26}{ }^{k} y_{27} y_{20}$ $x_{38}{ }^{i} x_{48}{ }^{j} x_{54}{ }^{h}$ are non-trivial and they and $x_{28}{ }^{k} a_{9} A$ (if it is non-trivial) are linearly independent.

Comparing $x_{28}{ }^{k} a_{9} A$ again with $d f_{2 k}$, we see that $x_{20}{ }^{k} a_{9} A$ is in the $d$-image only when $A=\partial P$, and then $x_{28}{ }^{k} a_{9} \partial P=d\left(x_{26}{ }^{k} P\right)$. Referring to (5.12.3), we see that $x_{28}{ }^{k} a_{9} x_{36}{ }^{i} x_{48}{ }^{j} x_{54}{ }^{h}, \quad x_{26}{ }^{k} a_{9} y_{21} x_{36}{ }^{i} x_{48}{ }^{j} x_{54}{ }^{h}, \quad x_{28}{ }^{k} a_{9} y_{22} x_{36}{ }^{i} x_{48}{ }^{j} x_{54}{ }^{h}, x_{26}{ }^{k} a_{9} y_{28} x_{36}{ }^{i} x_{48}{ }^{j}$ $x_{54}{ }^{h}$ and their sum are non-trivial.

Again by Lemma 5.14, a cocycle of degree $2 k+2$ is of the form $x_{26}{ }^{k+1} A$, where $A$ is a cocycle with neither $a_{9}$ nor $c_{17}$. Comparing $x_{26}{ }^{k+1} A$ with $d f_{2 k+1}$ as in the proof of Lemma 4 . 7, we see that $x_{26}{ }^{k+1} A$ is in the $d$-image only when $A=\partial^{2}(-Q)$, and then

$$
x_{26}{ }^{k+1} \partial^{2}(-Q)=d\left(x_{26}{ }^{k}\left(a_{9} Q+c_{17} \partial Q\right)\right) .
$$

By $(5.12 .1) \sim(5.12 .3)$ we see that

$$
\begin{aligned}
& x_{28}{ }^{k} x_{38}{ }^{i} x_{48}{ }^{j} x_{54}{ }^{h}, \quad x_{28}{ }^{k} a_{4} x_{36}{ }^{i} x_{48}{ }^{j} x_{54}{ }^{h}, x_{28}{ }^{k} a_{8} x_{36}{ }^{i} x_{48}{ }^{j} x_{54}{ }^{h}, \quad x_{28}{ }^{k} a_{10} x_{38}{ }^{i} x_{48}{ }^{j} x_{54}{ }^{h}, \\
& x_{28}{ }^{k} y_{20} x_{38}{ }^{i} x_{48}{ }^{j} x_{54}{ }^{h}, x_{28}{ }^{k} y_{22} x_{38}{ }^{i} x_{48}{ }^{j} x_{54}{ }^{h}, x_{28}{ }^{k} y_{28} x_{38}{ }^{i} x_{48}{ }^{j} x_{54}{ }^{h}, x_{28}{ }^{k} a_{10} y_{20} x_{38}{ }^{i} x_{48}{ }^{j} x_{54}{ }^{h}
\end{aligned}
$$

and their sum are non-trivial.

We have shown that the elements in the lemma are non-trivial and that they are linearly independent.

q.e.d.

Finally,

Lemma 5. 18. A cocycle with $a_{9}$ and $c_{17}$ is either trivial or a linear combination of the cocycles in Lemma 5.17.

Proof. Recall from (4.6.1) and (5.15.1) the relations

$$
a_{9}{ }^{2}=d c_{17}, \quad y_{21}{ }^{2}=d\left(c_{17} b_{12}{ }^{2}\right), \quad y_{25}{ }^{2}=d\left(c_{17} b_{18}{ }^{2}\right), \quad y_{27}{ }^{2}=d\left(c_{17} b_{18}{ }^{2}\right),
$$$$
a_{9} y_{21}=-x_{28} a_{4}+d\left(c_{17} b_{12}\right), \quad a_{9} y_{25}=-x_{28} a_{8}+d\left(c_{17} b_{18}\right), a_{9} y_{27}=-x_{28} a_{10}+d\left(c_{17} b_{18}\right),
$$$$
y_{21} y_{25}=-x_{28} y_{20}+d\left(c_{17} b_{12} b_{16}\right), y_{21} y_{27}=x_{28} y_{22}+d\left(c_{17} b_{12} b_{18}\right), y_{25} y_{27}=x_{28} y_{28}+d\left(c_{17} b_{18} b_{18}\right) \text {. }
$$

Therefore, any monomial in cocycles is reduced to a monomial that has at most one of $a_{9}, y_{21}, y_{25}$ or $y_{27}$.

We have shown that

(5. 19. 1) $\quad x_{26} \partial^{2} Q=d\left(-a_{9} Q-c_{17} \partial Q\right)$,

and $a_{9} \partial Q=d Q$, in particular, 
(5. 19. 2) $a_{9} \partial^{2} Q=d(\partial Q), a_{9} a_{4}=d\left(-b_{12}\right), a_{9} a_{8}=d\left(-b_{16}\right), a_{9} a_{10}=d\left(-b_{18}\right)$.

Finally we have the following $d$-images:

(5. 19. 3)

$$
\begin{array}{ll}
y_{21} \partial^{2} Q=d\left(a_{4} Q+b_{12} \partial Q\right), & y_{25} \partial^{2} Q=d\left(a_{8} Q+b_{18} \partial Q\right), \\
y_{27} \partial^{2} Q=d\left(a_{10} Q+b_{18} \partial Q\right), & y_{25} a_{8}=d\left(b_{18}{ }^{2}\right), \\
y_{21} a_{4}=d\left(b_{12}{ }^{2}\right), & y_{27} a_{10}=d\left(b_{18}{ }^{2}\right), \\
y_{21} a_{8}+a_{9} y_{20}=y_{25} a_{4}-a_{9} y_{20}=d\left(b_{12} b_{18}\right), & \\
y_{21} a_{10}-a_{9} y_{22}=y_{27} a_{4}+a_{9} y_{22}=d\left(b_{12} b_{18}\right), & \\
y_{25} a_{10}-a_{9} y_{26}=y_{27} a_{8}+a_{9} y_{28}=d\left(b_{18} b_{28}\right), \\
y_{21} y_{20}=d\left(-b_{12}{ }^{2} b_{18}\right), & y_{25} y_{20}=d\left(b_{12} b_{18}{ }^{2}\right), \\
y_{21} y_{22}=d\left(b_{12}{ }^{2} b_{18}\right), & y_{27} y_{22}=d\left(-b_{12} b_{18}{ }^{2}\right), \\
y_{25} y_{28}=d\left(b_{18}{ }^{2} b_{18}\right), & y_{27} y_{26}=d\left(-b_{18} b_{18}{ }^{2}\right), \\
y_{27} y_{20}-y_{25} y_{22}=y_{25} y_{22}+y_{21} y_{28}=-y_{27} y_{20}-y_{21} y_{28}=d\left(-b_{12} b_{18} b_{18}\right) .
\end{array}
$$

Using these relations we see that any monomial in cocycles with $a_{9}$ and $c_{17}$ is either trivial or equivalent to one of the elements in Lemma 5.17.

q.e.d.

Thus we have

Theorem 5. 20. For $B=H^{*}\left(X_{6} ; Z_{3}\right)$, we have as algebra

$\operatorname{Cotor}_{B}\left(\boldsymbol{Z}_{3}, \boldsymbol{Z}_{3}\right) \cong \boldsymbol{Z}_{3}\left[a_{9}, x_{28}, y_{21}, y_{25}, y_{27}, a_{4}, a_{8}, a_{10}, x_{38}, x_{48}, x_{54}\right.$, $\left.y_{20}, y_{22}, y_{28}, y_{58}, y_{80}, y_{64}, y_{78}\right] / \eta$,

where $\eta$ is the ideal generated by

i) elements which are 0 as polynomial in $Z_{3}\left[a_{4}, a_{8}, a_{10}, b_{12}, b_{18}, b_{18}\right]$;

ii) $a_{9}{ }^{2}, y_{21}{ }^{2}, y_{25}{ }^{2}, y_{27}{ }^{2}$,

$a_{9} y_{21}+x_{28} a_{4}, \quad a_{9} y_{25}+x_{28} a_{8}, \quad a_{9} y_{27}+x_{28} a_{10}$,

$y_{21} y_{25}+x_{28} y_{20}, y_{21} y_{27}-x_{28} y_{22}, y_{25} y_{27}-x_{28} y_{28}$,

iii) $a_{9} \partial^{2} Q, y_{21} \partial^{2} Q, y_{25} \partial^{2} Q, y_{27} \partial^{2} Q, x_{28} \partial^{2} Q$,

$a_{9} a_{4}, a_{9} a_{8}, a_{9} a_{10}$,

$y_{21} a_{4}, y_{25} a_{8}, y_{27} a_{10}$,

$y_{21} a_{8}+a_{9} y_{20}=y_{25} a_{4}-a_{9} y_{20}$,

$y_{21} a_{10}-a_{9} y_{22}=y_{27} a_{4}+a_{9} y_{22}$,

$y_{25} a_{10}-a_{9} y_{28}=y_{27} a_{8}+a_{9} y_{28}$,

$y_{21} y_{20}, y_{25} y_{20}, y_{21} y_{22}, y_{27} y_{22}, y_{25} y_{28}, y_{27} y_{28}$,

$y_{27} y_{20}-y_{25} y_{22}=y_{25} y_{22}+y_{21} y_{20}=-y_{27} y_{20}-y_{21} y_{26}$. 
(See Result (v)' and Propositions 5.10 and 5.13 for the expression of the generators in terms of $a_{i}{ }^{\prime} s, b_{j}{ }^{\prime} s$ and $c_{17}$. See also Remark 5.12 for practical use of the $\partial^{2}$-image.)

\author{
INSTITUTE OF MATHEMATICS \\ YOSHIDA COLLEGE \\ KYOTO UNIVERSITY; \\ FACULTY OF MANAGEMENT \\ SENSHU UNIVERSITY
}

\title{
References
}

[1] S. Araki: On the non-commutativity of Pontrjagin rings mod 3 of some compact exceptional groups, Nagoya Math. J., 17 (1960), 225-260.

[2] E. H. Brown Jr.: Twisted tensor product I, Ann. Math., 69 (1959), 223-246.

[ 3 ] A. Iwai - A. Shimada: A remark on resolutions for Hopf algebras, Publ. RIMS of Kyoto Univ., 1 (1966), 187-198.

[4] A Kono: Hopf algebra structure of simple Lie groups, J. Math. Kyoto Univ., 17 (1977), 259-298.

[5] A. Kono - M. Mimura: Cohomology mod 2 of the classifying space of the compact connected Lie group of type $E_{6}$, J. Pure and Applied Algebra, 6 (1975), 61-81.

[6] A. Kono - M. Mimura: Cohomology mod 3 of the classifying space of the compact, 1 connected Lie group of type $E_{8}$, Preprint Series 1974/75 No. 30., Matematisk Institut, Aarhus Universitet.

[7] A. Kono - M. Mimura: Cohomology operations and the Hopf algebra structure of the compact exceptional Lie groups $E_{7}$ and $E_{8}$, Proc. London Math. Soc., (3) 35 (1977), 345-358.

[8] A. Kono - M. Mimura - N.Shimada: Cohomology of classifying space of certain associative $H$-spaces, J. Math. Kyoto Univ., 15 (1975), 607-617.

[9] A. Kono-M. Mimura - N. Shimada: On the cohomology $\bmod 2$ of the classifying space of the 1-connected exceptional Lie group $E_{7}$, J. Pure and Applied Algebra, 8 (1976), 267-283.

[10] M. Mori: A note on Steenrod operations in the Eilenberg-Moore spectral sequence, Proc. Japan Acad., 53 (1977), 112-114.

[11] M. Mori: The Steenrod operations in the Eilenberg-Moore spectral sequence, Hiroshima Math. J., 9 (1979), 17-34.

[12] M. Rothenberg - N. E. Steenrod: The cohomology of classifying spaces of $H$-spaces, Bull. AMS, 71 (1965), 872-875.

[13] M. Rothenberg - N. E. Steenrod: The cohomology of classifying spaces of $H$-spaces, (mimeographed notes).

[14] N. Shimada - A. Iwai: On the cohomology of some Hopf algebra, Nagoya Math. J., 30 (1971), 103-111. 